\title{
Approximation of Holomorphic Mappings on Infinite Dimensional Spaces
}

\author{
Erhan ÇALıSTKAN \\ Departamento de Matemática e Estatística \\ Universidade Federal de Campina Grande \\ Caixa Postal 10044 \\ 58109-970 Campina Grande, PB, Brazil \\ caliskan@dme.ufcg.edu.br
}

Recibido: 1 de Diciembre de 2003

Aceptado: 26 de Abril de 2004

\begin{abstract}
In this article we examine necessary and sufficient conditions for the predual of the space of holomorphic mappings of bounded type, $G_{b}(U)$, to have the approximation property and the compact approximation property and we consider when the predual of the space of holomorphic mappings, $G(U)$, has the compact approximation property. We obtain also similar results for the preduals of spaces of $m$-homogeneous polynomials, $Q\left({ }^{m} E\right)$.
\end{abstract}

Key words: Holomorphic mappings; holomorphic mappings of bounded type; approximation property; compact approximation property.

2000 Mathematics Subject Classification: 46G20, 46B28, 46E50.

\section{Introduction}

Several authors have studied the connections between approximation properties on a space $E$ and approximation properties on the spaces of holomorphic functions on open subsets of $E$ and their preduals. We mention R. Aron and M. Schottenloher [3], M. Schottenloher [30], J. Mujica [26], [27], C. Boyd [9], C. Boyd, S. Dineen and P. Rueda [10], S. Dineen and J. Mujica [13], and the author [11]. The aim of this work is to investigate the relationships between the approximation property and the compact approximation property on a space $E$ and approximation properties on the spaces of holomorphic mappings $\mathcal{H}(U ; F)$ and $\mathcal{H}^{\infty}(\mathcal{U} ; F)$. 
This paper is organized as follows. In Section 2 we establish several properties of the locally convex space $\mathcal{H}^{\infty}(\mathcal{U} ; F)$ and the predual $G^{\infty}(\mathcal{U})$, which will be useful in subsequent sections.

In Section 3 we show that a Banach space $E$ has the approximation property if and only if $G^{\infty}(\mathcal{U})$ has the approximation property if and only if every $f \in \mathcal{H}^{\infty}(\mathcal{U} ; F)$ can be approximated by holomorphic mappings of finite rank for the topology $\tau_{\gamma}$ introduced in Section 2. This gives a new proof and also improves a result of J. Mujica [27, Theorem 4.2].

Section 4 is devoted to the study of the compact approximation property in locally convex spaces.

In Section 5 we show that a Banach space $E$ has the compact approximation property if and only if $G^{\infty}(\mathcal{U})$ has the compact approximation property if and only if every $f \in \mathcal{H}^{\infty}(\mathcal{U} ; F)$ can be approximated by holomorphic mappings with relatively compact range for the topology $\tau_{\gamma}$.

Finally in Section 6 we show that a Fréchet space $E$ has the compact approximation property if and only if $\left(\mathcal{H}(U), \tau_{c}\right)$ has the compact approximation property if and only if $G(U)$ has the compact approximation property. This parallels a result of C. Boyd [9].

\section{The spaces $\mathcal{H}^{\infty}(\mathcal{U} ; F)$ and $G^{\infty}(\mathcal{U})$}

The letter $\mathbb{C}$ denotes the field of all complex numbers, $\mathbb{N}$ denotes the set of all positive integers, and $\mathbb{N}_{0}=\mathbb{N} \cup\{0\}$.

Unless stated otherwise $E$ and $F$ denote locally convex spaces, always assumed complex and Hausdorff. The letter $U$ will always denote a nonvoid open subset of $E$. $\mathcal{V}_{0}(E)$ denotes the collection of neighborhoods of zero in $E . \Gamma(A)$ denotes the convex, balanced hull of a set $A \subset E$.

Given a topology $\tau$ on a set $E, \bar{N}^{\tau}$ denotes the $\tau$-closure of a set $N \subset E$.

Let $\mathcal{H}(U ; F)$ denote the vector space of all holomorphic mappings from $U$ into $F$, and let $\mathcal{H}^{\infty}(U ; F)$ denote the space of all $f \in \mathcal{H}(U ; F)$ such that $f(U)$ is bounded in $F . \mathcal{H}_{b}(U ; F)$ denotes the space of all $f \in \mathcal{H}(U ; F)$ such that $f(A)$ is bounded in $F$ for each $U$-bounded set $A$. We recall that a set $A \subset U$ is said to be $U$-bounded if $A$ is bounded and there exists $V \in \mathcal{V}_{0}(E)$ such that $A+V \subset U$.

Let $\mathcal{U}=\left(U_{n}\right)_{n \in \mathbb{N}}$ be an increasing countable open cover of $U$. Let $\mathcal{H}^{\infty}(\mathcal{U} ; F)$ denote the locally convex space

$$
\mathcal{H}^{\infty}(\mathcal{U} ; F)=\left\{f \in \mathcal{H}(U ; F): f\left(U_{n}\right) \text { is bounded in } F \text { for every } n\right\},
$$

endowed with the topology of uniform convergence on all the sets $U_{n}$.

It is clear that $\mathcal{H}_{b}(U ; F)=\mathcal{H}^{\infty}(\mathcal{U} ; F)$ if $\mathcal{U}=\left(U_{n}\right)_{n \in \mathbb{N}}$ is a fundamental sequence of open $U$-bounded sets. If $F=\mathbb{C}$ then we denote $\mathcal{H}(U ; \mathbb{C})\left(\operatorname{resp} . \mathcal{H}^{\infty}(U ; \mathbb{C}), \mathcal{H}_{b}(U ; \mathbb{C})\right.$, $\left.\mathcal{H}^{\infty}(\mathcal{U} ; \mathbb{C})\right)$ by $\mathcal{H}(U)\left(\operatorname{resp} . \mathcal{H}^{\infty}(U), \mathcal{H}_{b}(U), \mathcal{H}^{\infty}(\mathcal{U})\right)$

One can readily see that the space $\mathcal{H}^{\infty}(\mathcal{U} ; F)$ is canonically topologically isomorphic to the projective limit of the spaces $\mathcal{H}^{\infty}\left(U_{n} ; F\right)$. Likewise, if $\tau_{c}$ denotes the 
compact open topology, then the space $\left(\mathcal{H}^{\infty}(\mathcal{U} ; F), \tau_{c}\right)$ is canonically topologically isomorphic to the projective limit of the spaces $\left(\mathcal{H}^{\infty}\left(U_{n} ; F\right), \tau_{c}\right)$. In each case the isomorphism is given by the mapping

$$
f \in \mathcal{H}^{\infty}(\mathcal{U} ; F) \longrightarrow\left(\left.f\right|_{U_{n}}\right)_{n=1}^{\infty} \in \underset{n \in \mathbb{N}}{\operatorname{proj}} \mathcal{H}^{\infty}\left(U_{n} ; F\right) .
$$

We refer to [12] and [25] for the properties of holomorphic mappings in infinite dimensional spaces, to [17], [18], [19] and [20] for the theory of locally convex spaces, and to [5], [12], [17], [18] and [19] for the properties of projective and inductive limits.

We now introduce a very useful intermediate topology.

Definition 2.1. Let $E$ and $F$ be locally convex spaces, and let $U$ be an open subset of $E$.

(i) We denote by $\tau_{\gamma}$ the locally convex topology on $\mathcal{H}^{\infty}(U ; F)$ generated by all the seminorms of the form

$$
p(f)=\sup _{j} \alpha_{j} q\left(f\left(x_{j}\right)\right), \quad f \in \mathcal{H}^{\infty}(U ; F)
$$

where $\left(x_{j}\right)_{j=1}^{\infty}$ varies over all sequences in $U,\left(\alpha_{j}\right)_{j=1}^{\infty}$ varies over all sequences of positive numbers tending to zero, and $q$ varies over all continuous seminorms on $F$.

(ii) If $\mathcal{U}=\left(U_{n}\right)_{n \in \mathbb{N}}$ is an increasing, countable open cover of $U$ then we denote by $\tau_{\gamma}$ the locally convex topology on $\mathcal{H}^{\infty}(\mathcal{U} ; F)$ generated by all the seminorms of the form

$$
p(f)=\sup _{j} \alpha_{j} q\left(f\left(x_{j}\right)\right), f \in \mathcal{H}^{\infty}(\mathcal{U} ; F)
$$

where $\left(x_{j}\right)_{j=1}^{\infty}$ varies over all sequences in $U$ which are contained in some $U_{n}$, $\left(\alpha_{j}\right)_{j=1}^{\infty}$ varies over all sequences of positive numbers tending to zero, and $q$ varies over all continuous seminorms on $F$.

When $F$ is a Banach space, the topology $\tau_{\gamma}$ on $\mathcal{H}^{\infty}(U ; F)$ coincides with the topology $\tau_{\gamma}$ introduced by J. Mujica in [26]. One can also prove that the space $\left(\mathcal{H}^{\infty}(\mathcal{U} ; F), \tau_{\gamma}\right)$ is canonically topologically isomorphic to the projective limit of the spaces $\left(\mathcal{H}^{\infty}\left(U_{n} ; F\right), \tau_{\gamma}\right)$ with the same isomorphism as above.

Let $\mathcal{P}(E ; F)$ denote the vector space of all continuous polynomials from $E$ into $F$ and let $\mathcal{P}\left({ }^{m} E ; F\right)$ denote the subspace of all $m$-homogeneous members of $\mathcal{P}(E ; F)$ for every $m \in \mathbb{N}_{0}$. In case $F=\mathbb{C}$ we denote $\mathcal{P}\left({ }^{m} E ; \mathbb{C}\right)$ by $\mathcal{P}\left({ }^{m} E\right)$.

Proposition 2.2. Let $E$ be a Banach space, let $F$ be a complete locally convex space, let $U$ be an open subset of $E$, and let $\mathcal{U}=\left(U_{n}\right)_{n \in \mathbb{N}}$ be an increasing countable open cover of $U$ such that every $U_{n}$ is bounded and balanced. Then

(i) $\tau_{\gamma} \geq \tau_{c}$ on $\mathcal{H}^{\infty}(\mathcal{U} ; F)$. 
(ii) $\tau_{\gamma}=\tau_{c}$ on $\mathcal{P}\left({ }^{m} E ; F\right)$ for every $m \in \mathbb{N}$.

Proof. (i) As remarked above $\tau_{c}$ is the weakest topology on $\mathcal{H}^{\infty}(\mathcal{U} ; F)$ such that each restriction $R_{n}:\left(\mathcal{H}^{\infty}(\mathcal{U} ; F), \tau_{c}\right) \longrightarrow\left(\mathcal{H}^{\infty}\left(U_{n} ; F\right), \tau_{c}\right)$ is continuous. Let us consider the following commutative diagram:

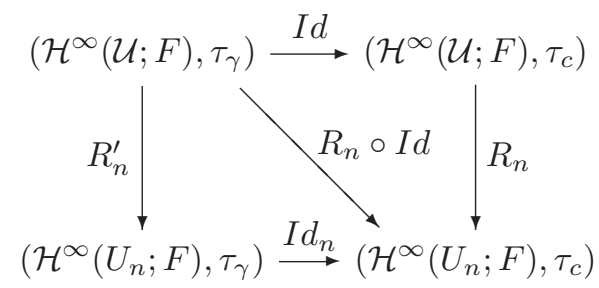

where $I d$ and $I d_{n}$ are identity mappings on $\mathcal{H}^{\infty}(\mathcal{U} ; F)$ and $\mathcal{H}^{\infty}\left(U_{n} ; F\right)$, respectively. By the definition of $\tau_{\gamma}$ and by [26, Proposition 4.9] and by the fact that a complete locally convex space is the projective limit of Banach spaces, for every $n \in \mathbb{N}$ the composition $I d_{n} \circ R_{n}^{\prime}$ is continuous. Since $R_{n} \circ I d=I d_{n} \circ R_{n}^{\prime}$, then $R_{n} \circ I d$ is continuous for every $n \in \mathbb{N}$. Hence $I d$ is continuous. Thus $\tau_{\gamma} \geq \tau_{c}$ on $\mathcal{H}^{\infty}(\mathcal{U} ; F)$.

(ii) Let $m \in \mathbb{N}$. From (i) we have that $\tau_{\gamma} \geq \tau_{c}$ on $\mathcal{P}\left({ }^{m} E ; F\right)$. Now we show that $\tau_{\gamma} \leq \tau_{c}$ on $\mathcal{P}\left({ }^{m} E ; F\right)$. Let $n \in \mathbb{N}$ and let us consider the following commutative diagram:

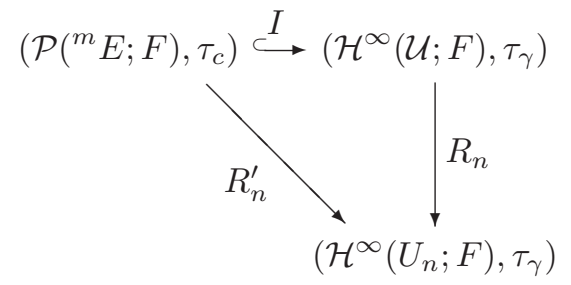

Let $q$ be continuous seminorm on $\left(\mathcal{H}^{\infty}\left(U_{n} ; F\right), \tau_{\gamma}\right)$. For every $P \in \mathcal{P}\left({ }^{m} E ; F\right)$ we have

$$
q \circ R_{n}^{\prime}(P)=q\left(\left.P\right|_{U_{n}}\right)=\sup _{j} \alpha_{j} p\left(\left.P\right|_{U_{n}}\left(x_{j}\right)\right)=\sup _{j} p\left(P\left(\left(\alpha_{j}\right)^{\frac{1}{m}} x_{j}\right)\right),
$$

where $\left(x_{j}\right)_{j=1}^{\infty} \subset U_{n},\left(\alpha_{j}\right)_{j=1}^{\infty}$ is a sequence of positive numbers tending to zero and $p$ is a continuous seminorm on $F$. Let $\beta_{j}=\sqrt[m]{\alpha_{j}}$ for every $j \in \mathbb{N}$ and let $K=\left\{\beta_{j} x_{j}: j \in \mathbb{N}\right\} \cup\{0\}$. Since $U_{n}$ is bounded, $\lim _{j \rightarrow \infty} \beta_{j} x_{j} \longrightarrow 0$. Thus $K$ is compact in $U_{n}$. Therefore $\sup _{j} p\left(P\left(\beta_{j} x_{j}\right)\right)$ defines a continuous seminorm on $\left(\mathcal{P}\left({ }^{m} E ; F\right), \tau_{c}\right)$. Hence $R_{n}^{\prime}$ is continuous. Since for every $n \in \mathbb{N}, R_{n}^{\prime}=R_{n} \circ I$, then the inclusion mapping $I$ is continuous. Thus $\tau_{\gamma} \leq \tau_{c}$ on $\mathcal{P}\left({ }^{m} E ; F\right)$. 
Let $L(E ; F)$ denote the vector space of all continuous linear mappings from $E$ into $F$. We say that $T \in L(E ; F)$ is a compact mapping if there is some neighborhood $V$ of zero in $E$ such that $T(V)$ is relatively compact in $F$. We denote by $L_{k}(E ; F)$ the vector subspace of all compact mappings of $L(E ; F)$.

P. Galindo, D. Garcia and M. Maestre [15] constructed a complete (LB)-space $G_{b}(U)$ and a mapping $\delta_{U} \in \mathcal{H}_{b}\left(U ; G_{b}(U)\right)$ with the following universal property: For each Banach space $F$ and each mapping $f \in \mathcal{H}_{b}(U ; F)$, there is a unique mapping $T_{f} \in L\left(G_{b}(U) ; F\right)$ such that $T_{f} \circ \delta_{U}=f$. J. Mujica [27] generalized this result as follows:

Theorem 2.3 ([27, Theorem 2.1]). Let $U$ be an open subset of a locally convex space $E$, and let $\mathcal{U}=\left(U_{n}\right)_{n \in \mathbb{N}}$ be an increasing countable open cover of $U$. Then there are a complete $(L B)$-space $G^{\infty}(\mathcal{U})$ and a mapping $\delta_{\mathcal{U}} \in \mathcal{H}^{\infty}\left(\mathcal{U} ; G^{\infty}(\mathcal{U})\right.$ ) with the following universal property: For each complete locally convex space $F$ and each mapping $f \in \mathcal{H}^{\infty}(\mathcal{U} ; F)$, there is a unique mapping $T_{f} \in L\left(G^{\infty}(\mathcal{U}) ; F\right)$ such that $T_{f} \circ$ $\delta_{\mathcal{U}}=f$. This property characterize $G^{\infty}(\mathcal{U})$ uniquely up to a topological isomorphism.

The space $G^{\infty}(\mathcal{U})$ is defined in the following way: For every sequence $\alpha=\left(\alpha_{n}\right)$ of strictly positive numbers, let $B_{\mathcal{U}}^{\alpha}=\left\{f \in \mathcal{H}^{\infty}(\mathcal{U}):\|f\|_{U_{n}} \leq \alpha_{n}\right.$ for every $\left.n\right\}$. Then we define $G^{\infty}(\mathcal{U})=\left\{u \in \mathcal{H}^{\infty}(\mathcal{U})^{\prime}:\left.u\right|_{B_{\mathcal{U}}^{\alpha}}\right.$ is $\tau_{c^{-}}$continuous for every $\left.\alpha\right\}$, endowed with the topology of uniform convergence on all the sets $B_{\mathcal{U}}^{\alpha}$. The evaluation mapping $\delta_{\mathcal{U}}: x \in U \longrightarrow \delta_{x} \in G^{\infty}(\mathcal{U})$ is defined by $\delta_{x}: f \in \mathcal{H}^{\infty}(\mathcal{U}) \longrightarrow f(x) \in \mathbb{C}$ for every $x \in U$. As in the case of bounded holomorphic mappings the evaluations $\delta_{x}$, $x \in U$, generate a dense vector subspace of $G^{\infty}(\mathcal{U})$ (see [27, Theorem 2.1] and [28, Theorem 2.1].

Proposition 2.4. Let $E$ be a Banach space, let $U$ be an open balanced subset of $E$, and let $\mathcal{U}=\left(U_{n}\right)_{n \in \mathbb{N}}$ be a sequence of bounded, balanced, open subsets of $U$ such that $U=\bigcup_{n=1}^{\infty} U_{n}$ and $\rho_{n} U_{n} \subset U_{n+1}$, with $\rho_{n}>1$, for every $n \in \mathbb{N}$. Then we have the following algebraic isomorphisms:

(i) $L\left(G^{\infty}(\mathcal{U}) ; F\right) \cong \operatorname{proj}_{n \in \mathbb{N}} L\left(G^{\infty}\left(U_{n}\right) ; F\right)$ for every locally convex space $F$.

(ii) $L_{k}\left(G^{\infty}(\mathcal{U}) ; F\right) \cong \operatorname{proj}_{n \in \mathbb{N}} L_{k}\left(G^{\infty}\left(U_{n}\right) ; F\right)$ for every Fréchet space $F$.

Proof. (i) It follows from [27, Theorem 3.2] and [23, §1, Satz 3].

(ii) If $T \in L_{k}\left(G^{\infty}(\mathcal{U}) ; F\right)$, then there is a $V \in \mathcal{V}_{0}\left(G^{\infty}(\mathcal{U})\right)$ such that $T(V)$ is relatively compact in $F$. Thus $i_{n}^{-1}(V) \in \mathcal{V}_{0}\left(G^{\infty}\left(U_{n}\right)\right)$, where for every $n \in \mathbb{N}$, $i_{n}: G^{\infty}\left(U_{n}\right) \hookrightarrow G^{\infty}(\mathcal{U})$ is the inclusion mapping. Hence for the corresponding mapping $\left(\left.T\right|_{G^{\infty}\left(U_{n}\right)}\right)_{n \in \mathbb{N}} \in \operatorname{proj}_{n \in \mathbb{N}} L\left(G^{\infty}\left(U_{n}\right) ; F\right)$ we have $\overline{\left.T\right|_{G^{\infty}\left(U_{n}\right)}\left(i_{n}^{-1}(V)\right)} \subset$ $\overline{T(V)}$ for every $n \in \mathbb{N}$, which shows that

$$
\left(\left.T\right|_{G^{\infty}\left(U_{n}\right)}\right)_{n \in \mathbb{N}} \in \underset{n \in \mathbb{N}}{\operatorname{proj}} L_{k}\left(G^{\infty}\left(U_{n}\right) ; F\right) .
$$


Conversely let $\left(T_{n}\right)_{n \in \mathbb{N}} \in \operatorname{proj}_{n \in \mathbb{N}} L_{k}\left(G^{\infty}\left(U_{n}\right) ; F\right)$. We show that there is a $W \in \mathcal{V}_{0}\left(G^{\infty}(\mathcal{U})\right)$ such that $T(W)$ is relatively compact in $F$, where the corresponding mapping $T$ is defined by $T(x)=T_{n}(x)$ for every $x \in G^{\infty}\left(U_{n}\right)$. By the hypotheses for every $n \in \mathbb{N}$ there is $U_{n} \in \mathcal{V}_{0}\left(G^{\infty}\left(U_{n}\right)\right)$ such that $T_{n}\left(U_{n}\right)$ is relatively compact in $F$. Hence by [24, Lemma 7.8] there is a sequence $\left(c_{n}\right)_{n \in \mathbb{N}}$ of strictly positive numbers such that $\bigcup_{n=1}^{\infty} c_{n} \overline{T_{n}\left(U_{n}\right)}$ is relatively compact in $F$. By [27, Theorem 3.2] the set $W:=\Gamma\left(\bigcup_{n=1}^{\infty} i_{n}\left(c_{n} U_{n}\right)\right)$ is a neighborhood of zero in $G^{\infty}(\mathcal{U})$ (see, for example, [17, pg. 157]), and we have that

$$
T(W)=\Gamma\left(\bigcup_{n=1}^{\infty} T \circ i_{n}\left(c_{n} U_{n}\right)\right)=\Gamma\left(\bigcup_{n=1}^{\infty} T_{n}\left(c_{n} U_{n}\right)\right) \subset \Gamma\left(\overline{\bigcup_{n=1}^{\infty} c_{n} \overline{T_{n}\left(U_{n}\right)}}\right)
$$

Since $\Gamma\left(\overline{\left.\bigcup_{n=1}^{\infty} c_{n} \overline{T_{n}\left(U_{n}\right)}\right)}\right.$ is relatively compact in $F$, so is $T(W)$.

Proposition 2.5. Let $E$ be a Banach space, let $F$ be a complete locally convex space, let $U$ be an open balanced subset of $E$, and let $\mathcal{U}=\left(U_{n}\right)_{n \in \mathbb{N}}$ be a sequence of bounded, balanced, open subsets of $U$ such that $U=\bigcup_{n=1}^{\infty} U_{n}$ and $\rho_{n} U_{n} \subset U_{n+1}$, with $\rho_{n}>1$, for every $n \in \mathbb{N}$. Then

(i) $\left(L\left(G^{\infty}(\mathcal{U}) ; F\right), \tau_{c}\right)$ is topologically isomorphic to $\operatorname{proj}_{n \in \mathbb{N}}\left(L\left(G^{\infty}\left(U_{n}\right) ; F\right), \tau_{c}\right)$.

(ii) $\left(\mathcal{H}^{\infty}(\mathcal{U} ; F), \tau_{\gamma}\right)$ is topologically isomorphic to $\left(L\left(G^{\infty}(\mathcal{U}) ; F\right), \tau_{c}\right)$.

Proof. (i) By Proposition 2.4, $L\left(G^{\infty}(\mathcal{U}) ; F\right)$ and $\operatorname{proj}_{n \in \mathbb{N}} L\left(G^{\infty}\left(U_{n}\right) ; F\right)$ are algebraically isomorphic. Since, by $[27$, Theorem 3.2$]$, the inductive limit $\operatorname{ind}_{n \in \mathbb{N}} G^{\infty}\left(U_{n}\right)$ is boundedly retractive, it is compactly regular. Hence every compact set of $G^{\infty}(\mathcal{U})$ is contained and compact in $G^{\infty}\left(U_{n}\right)$ for some $n \in \mathbb{N}$. Conversely, since every $G^{\infty}\left(U_{n}\right)$ is a vector subspace of $G^{\infty}(\mathcal{U})$, and the inclusion $G^{\infty}\left(U_{n}\right) \hookrightarrow G^{\infty}(\mathcal{U})$ is continuous, then for every $n \in \mathbb{N}$, every compact set of $G^{\infty}\left(U_{n}\right)$ is a compact set of $G^{\infty}(\mathcal{U})$.

(ii) By the fact that a complete locally convex space is a projective limit of Banach spaces and by $\left[26\right.$, Theorem 4.8] $\left(\mathcal{H}^{\infty}\left(U_{n} ; F\right), \tau_{\gamma}\right) \cong\left(L\left(G^{\infty}\left(U_{n}\right) ; F\right), \tau_{c}\right)$ for every $n \in \mathbb{N}$, we have topological isomorphism $\operatorname{proj}_{n \in \mathbb{N}}\left(\mathcal{H}^{\infty}\left(U_{n} ; F\right), \tau_{\gamma}\right) \cong$ $\operatorname{proj}_{n \in \mathbb{N}}\left(L\left(G^{\infty}\left(U_{n}\right) ; F\right), \tau_{c}\right)$. As $\left(\mathcal{H}^{\infty}(\mathcal{U} ; F), \tau_{\gamma}\right) \cong \operatorname{proj}_{n \in \mathbb{N}}\left(\mathcal{H}^{\infty}\left(U_{n} ; F\right), \tau_{\gamma}\right)$, by (i) we have the isomorphism.

If $X$ is a set and $Y$ is a vector space, then a mapping $f: X \longrightarrow Y$ is said to have finite rank if the subspace $\langle f(X)\rangle$ generated by $f(X)$ is finite dimensional. The subspace of all mappings of finite rank of $\mathcal{H}(U ; F)$ can be canonically identified with $\mathcal{H}(U) \otimes F$. Hence $\mathcal{H}^{\infty}(\mathcal{U}) \otimes F$ denotes the subspace of all members of $\mathcal{H}^{\infty}(\mathcal{U} ; F)$ which have finite rank.

We denote by $\mathcal{H}_{K}^{\infty}(U ; F)$ the subspace of all members of $\mathcal{H}^{\infty}(U ; F)$ which have relatively compact range, and by $\mathcal{H}_{K}^{\infty}(\mathcal{U} ; F)$ the subspace of $\mathcal{H}^{\infty}(\mathcal{U} ; F)$ defined by

$\mathcal{H}_{K}^{\infty}(\mathcal{U} ; F)=\left\{f \in \mathcal{H}(U ; F): f\left(U_{n}\right)\right.$ is relatively compact in $F$ for every $\left.n\right\}$. 
Proposition 2.6. Let $E$ be a Banach space, let $U$ be an open connected subset of $E$, and let $\mathcal{U}=\left(U_{n}\right)_{n \in \mathbb{N}}$ be an increasing countable open cover of $U$. Then we have the following algebraic isomorphisms:

(i) $\mathcal{H}^{\infty}(\mathcal{U}) \otimes F \cong\left[\operatorname{proj}_{n \in \mathbb{N}} \mathcal{H}^{\infty}\left(U_{n}\right)\right] \otimes F \cong \operatorname{proj}_{n \in \mathbb{N}}\left[\mathcal{H}^{\infty}\left(U_{n}\right) \otimes F\right]$ for every locally convex space $F$.

(ii) $\mathcal{H}_{K}^{\infty}(\mathcal{U} ; F) \cong \operatorname{proj}_{n \in \mathbb{N}} \mathcal{H}_{K}^{\infty}\left(U_{n} ; F\right)$ for every locally convex space $F$.

Proof. Since (ii) is trivial we will prove (i). If $f \in \mathcal{H}^{\infty}(\mathcal{U}) \otimes F$ then clearly

$$
\left(\left.f\right|_{U_{n}}\right)_{n \in \mathbb{N}} \in \operatorname{proj}_{n \in \mathbb{N}}\left[\mathcal{H}^{\infty}\left(U_{n}\right) \otimes F\right] .
$$

To show the converse let $\left(f_{n}\right)_{n \in \mathbb{N}} \in \operatorname{proj}_{n \in \mathbb{N}}\left[\mathcal{H}^{\infty}\left(U_{n}\right) \otimes F\right]$. Then the corresponding mapping $f$, defined by $f(x)=f_{n}(x)$ for every $x \in U_{n}$, belongs to $\mathcal{H}^{\infty}(\mathcal{U}) \otimes F$. Indeed, since $f\left(U_{1}\right) \subset \overline{\left\langle f_{1}\left(U_{1}\right)\right\rangle}$, by [25, Exercise 5.F] we have that $f(U) \subset \overline{\left\langle f_{1}\left(U_{1}\right)\right\rangle}$. Thus $\operatorname{dim}\langle f(U)\rangle=\operatorname{dim}\left\langle f_{1}\left(U_{1}\right)\right\rangle$ and hence $f \in \mathcal{H}^{\infty}(\mathcal{U}) \otimes F$.

Proposition 2.7. Let $E$ be a Banach space, let $U$ be an open balanced subset of $E$, and let $\mathcal{U}=\left(U_{n}\right)_{n \in \mathbb{N}}$ be a sequence of bounded, balanced, open subsets of $U$ such that $U=\bigcup_{n=1}^{\infty} U_{n}$ and $\rho_{n} U_{n} \subset U_{n+1}$, with $\rho_{n}>1$, for every $n \in \mathbb{N}$. Then we have the following algebraic isomorphisms:

(i) $\mathcal{H}^{\infty}(\mathcal{U} ; F) \cong L\left(G^{\infty}(\mathcal{U}) ; F\right)$ for every complete locally convex space $F$.

(ii) $\mathcal{H}^{\infty}(\mathcal{U}) \otimes F \cong G^{\infty}(\mathcal{U})^{\prime} \otimes F$ for every complete locally convex space $F$.

(iii) $\mathcal{H}_{K}^{\infty}(\mathcal{U} ; F) \cong L_{k}\left(G^{\infty}(\mathcal{U}) ; F\right)$ for every Banach space $F$.

(iv) $G^{\infty}(\mathcal{U})^{\prime} \otimes F \cong\left[\operatorname{proj}_{n \in \mathbb{N}} G^{\infty}\left(U_{n}\right)^{\prime}\right] \otimes F \cong \operatorname{proj}_{n \in \mathbb{N}}\left[G^{\infty}\left(U_{n}\right)^{\prime} \otimes F\right]$ for every Banach space $F$.

Proof. (i) It follows from [27, Proposition 2.2].

(ii) By Theorem 2.3 we have that $f \in \mathcal{H}^{\infty}(\mathcal{U}) \otimes F$ if and only if $\operatorname{dim}\left\langle T_{f} \circ \delta_{\mathcal{U}}(U)\right\rangle<\infty$ if and only if $T_{f} \in G^{\infty}(\mathcal{U})^{\prime} \otimes F$.

(iii) If $f \in \mathcal{H}_{K}^{\infty}(\mathcal{U} ; F)$ then by Proposition 2.6 we have that $\left(\left.f\right|_{U_{n}}\right)_{n \in \mathbb{N}} \in$ $\operatorname{proj}_{n \in \mathbb{N}} \mathcal{H}_{K}^{\infty}\left(U_{n} ; F\right)$. Therefore by the part (i) and by [26, Proposition 3.4] we have the corresponding mapping $\left(T_{\left.f\right|_{U_{n}}}\right)_{n \in \mathbb{N}} \in \operatorname{proj}_{n \in \mathbb{N}} L_{k}\left(G^{\infty}\left(U_{n}\right) ; F\right)$ and by Proposition 2.4 the corresponding mapping $T_{f}$ belongs to $L_{k}\left(G^{\infty}(\mathcal{U}) ; F\right)$. By the same argument we can also show the converse.

(iv) By $[27$, Theorem 3.2] and $[14, \S 26$, Satz 1.2, pg. 142] we have that

$$
G^{\infty}(\mathcal{U})^{\prime} \otimes F \cong\left[\operatorname{proj}_{n \in \mathbb{N}} G^{\infty}\left(U_{n}\right)^{\prime}\right] \otimes F .
$$


Now we show that

$$
\underset{n \in \mathbb{N}}{\operatorname{proj}}\left[G^{\infty}\left(U_{n}\right)^{\prime} \otimes F\right] \cong G^{\infty}(\mathcal{U})^{\prime} \otimes F
$$

Since by (i) $\operatorname{proj}_{n \in \mathbb{N}} L\left(G^{\infty}\left(U_{n}\right) ; F\right) \cong \operatorname{proj}_{n \in \mathbb{N}} \mathcal{H}^{\infty}\left(U_{n} ; F\right)$, then by [26, Proposition 3.1] we have that $\operatorname{proj}_{n \in \mathbb{N}}\left[G^{\infty}\left(U_{n}\right)^{\prime} \otimes F\right] \cong \operatorname{proj}_{n \in \mathbb{N}}\left[\mathcal{H}^{\infty}\left(U_{n}\right) \otimes F\right]$. Furthermore, by Proposition 2.6, we have $\operatorname{proj}_{n \in \mathbb{N}}\left[\mathcal{H}^{\infty}\left(U_{n}\right) \otimes F\right] \cong \mathcal{H}^{\infty}(\mathcal{U}) \otimes$ $F$, and by (ii), we have $\mathcal{H}^{\infty}(\mathcal{U}) \otimes F \cong G^{\infty}(\mathcal{U})^{\prime} \otimes F$. Hence it follows that $\operatorname{proj}_{n \in \mathbb{N}}\left[G^{\infty}\left(U_{n}\right)^{\prime} \otimes F\right] \cong G^{\infty}(\mathcal{U})^{\prime} \otimes F$.

A polynomial $P \in \mathcal{P}\left({ }^{n} E ; F\right)$ is called of finite type if it is generated by linear combination of functions $\phi^{n} \otimes y\left(\phi \in E^{\prime}, y \in F\right)$, where $\phi^{n} \otimes y(x)=\phi^{n}(x) y$ for all $x \in E$. Let $\mathcal{P}_{f}\left({ }^{n} E ; F\right)$ denote the subspace of all members of $\mathcal{P}\left({ }^{n} E ; F\right)$ which are of finite type, for every $n \in \mathbb{N}_{0}$, and let $\mathcal{P}_{f}(E ; F)$ denote the subspace of all $P \in \mathcal{P}(E ; F)$ of the form $P=P_{0}+\cdots+P_{m}$, with $P_{j} \in \mathcal{P}_{f}\left({ }^{j} E ; F\right)$ for every $j$.

Proposition 2.8. Let $E$ be a Banach space, let $U$ be a balanced open subset of $E$, and let $\mathcal{U}=\left(U_{n}\right)_{n \in \mathbb{N}}$ be an increasing, countable open cover of $U$.

(i) If $\left(\mathcal{H}^{\infty}(\mathcal{U} ; F), \tau_{\gamma}\right)=\overline{\mathcal{H}^{\infty}(\mathcal{U}) \otimes F}$ for every Banach space $F$, then we have that

$$
\left(\mathcal{H}^{\infty}(\mathcal{U} ; F), \tau_{\gamma}\right)=\overline{\mathcal{H}^{\infty}(\mathcal{U}) \otimes F}
$$

for every locally convex space $F$.

(ii) If $\left(\mathcal{H}^{\infty}(\mathcal{U} ; F), \tau_{\gamma}\right)=\overline{\mathcal{P}_{f}(E ; F)}$ for every Banach space $F$, then we have that

$$
\left(\mathcal{H}^{\infty}(\mathcal{U} ; F), \tau_{\gamma}\right)=\overline{\mathcal{P}_{f}(E ; F)}
$$

for every locally convex space $F$.

Proof. We only will prove (i), and since $\mathcal{P}_{f}(E ; F)=\mathcal{P}_{f}(E) \otimes F$ the same proof works for (ii). Suppose that $\left(\mathcal{H}^{\infty}(\mathcal{U} ; G), \tau_{\gamma}\right)=\overline{\mathcal{H}^{\infty}(\mathcal{U}) \otimes G}$ for every Banach space $G$. Let $F$ be a locally convex space and let $V \in \mathcal{V}_{0}(F), V$ open, balanced and convex. Let $P_{V}$ be the Minkowski funcional of $V$, let $F_{V}$ be the normed space $F_{V}=\left(F, P_{V}\right) / P_{V}^{-1}(0)$ and let $\widetilde{F}_{V}$ be its completion. Then by hypothesis we have that $\left(\mathcal{H}^{\infty}\left(\mathcal{U} ; \widetilde{F}_{V}\right), \tau_{\gamma}\right)=$ $\overline{\mathcal{H}^{\infty}(\mathcal{U}) \otimes \widetilde{F}_{V}}$. Let $p$ be a continuous seminorm on $\left(\mathcal{H}^{\infty}\left(\mathcal{U} ; \widetilde{F}_{V}\right), \tau_{\gamma}\right)$. Without loss of generality we may assume that

$$
p(\tilde{f})=\sup _{i} \alpha_{i}\left\|\tilde{f}\left(x_{i}\right)\right\| \text { for all } \tilde{f} \in \mathcal{H}^{\infty}\left(\mathcal{U} ; \widetilde{F}_{V}\right)
$$

where $\left(x_{i}\right)_{i \in \mathbb{N}} \subset U_{n}$ for some $n \in \mathbb{N}$, and $\left(\alpha_{i}\right)_{i \in \mathbb{N}}$ is a sequence of positive numbers tending to zero. Let $f \in \mathcal{H}^{\infty}(\mathcal{U} ; F)$, let $\epsilon>0$ and let $P$ be a continuous seminorm on 
$\left(\mathcal{H}^{\infty}(\mathcal{U} ; F), \tau_{\gamma}\right)$. We want to find $g \in \mathcal{H}^{\infty}(\mathcal{U}) \otimes F$ such that $P(f-g) \leq \epsilon$. Without loss of generality we may assume that

$$
P(f)=\sup _{i} \alpha_{i} P_{V}\left(f\left(x_{i}\right)\right) \text { for all } f \in \mathcal{H}^{\infty}(\mathcal{U} ; F) .
$$

Let $\Pi_{V} \in L\left(F ; F_{V}\right)$ be the canonical mapping

$$
\Pi_{V}: F \longrightarrow F_{V} \subset \widetilde{F}_{V}
$$

Since $\Pi_{V} \circ f \in \mathcal{H}^{\infty}\left(\mathcal{U} ; \widetilde{F}_{V}\right)$, then there is a $\tilde{g} \in \mathcal{H}^{\infty}(\mathcal{U}) \otimes \widetilde{F}_{V}$ with $\tilde{g}(x)=\sum_{j=1}^{k} \varphi_{j}(x) b_{j}$ for every $x \in U$, where $\varphi_{j} \in \mathcal{H}^{\infty}(\mathcal{U})$ and $b_{j} \in \widetilde{F}_{V}$, such that

$$
p\left(\Pi_{V} \circ f-\tilde{g}\right)=\sup _{i} \alpha_{i}\left\|\Pi_{V} \circ f\left(x_{i}\right)-\tilde{g}\left(x_{i}\right)\right\|<\frac{\epsilon}{2} .
$$

Furthermore for each $j \in \mathbb{N}$ there is a $a_{j} \in F$ such that

$$
\left\|\Pi_{V}\left(a_{j}\right)-b_{j}\right\|<\frac{\epsilon}{2 k \sup _{i} \alpha_{i}\left|\varphi_{j}\left(x_{i}\right)\right|} .
$$

Thus for every $i \in \mathbb{N}$ we have that

$$
\begin{aligned}
& \left\|\Pi_{V}\left(\alpha_{i} f\left(x_{i}\right)-\alpha_{i} \sum_{j=1}^{k} \varphi_{j}\left(x_{i}\right) a_{j}\right)\right\|=\left\|\alpha_{i} \Pi_{V} \circ f\left(x_{i}\right)-\alpha_{i} \sum_{j=1}^{k} \varphi_{j}\left(x_{i}\right) \Pi_{V}\left(a_{j}\right)\right\| \\
& \quad \leq\left\|\alpha_{i} \Pi_{V} \circ f\left(x_{i}\right)-\alpha_{i} \sum_{j=1}^{k} \varphi_{j}\left(x_{i}\right) b_{j}\right\|+\left\|\alpha_{i} \sum_{j=1}^{k} \varphi_{j}\left(x_{i}\right)\left(b_{j}-\Pi_{V}\left(a_{j}\right)\right)\right\|<\epsilon .
\end{aligned}
$$

Hence for each $i \in \mathbb{N}$ we have that $\Pi_{V}\left(\alpha_{i} f\left(x_{i}\right)-\alpha_{i} \sum_{j=1}^{k} \varphi_{j}\left(x_{i}\right) a_{j}\right) \in \epsilon \Pi_{V}(V)$ since $\Pi_{V}(V)=U_{F_{V}}$, where $U_{F_{V}}$ is the unit open ball of $F_{V}$. Thus we get $\alpha_{i} f\left(x_{i}\right)-$ $\alpha_{i} \sum_{j=1}^{k} \varphi_{j}\left(x_{i}\right) a_{j} \in \epsilon \Pi_{V}^{-1}\left(\Pi_{V}(V)\right)=\epsilon V$ for each $i \in \mathbb{N}$. Hence defining a mapping of finite type $g: U \longrightarrow F$ with $g(x)=\sum_{j=1}^{k} \varphi_{j}(x) a_{j}$ for each $x \in U$, we obtain $\alpha_{i}\left(f\left(x_{i}\right)-g\left(x_{i}\right)\right) \in \epsilon V$ for each $i \in \mathbb{N}$, or equivalently, $\alpha_{i} P_{V}\left(f\left(x_{i}\right)-g\left(x_{i}\right)\right) \leq \epsilon$ for each $i \in \mathbb{N}$. Thus $P(f-g)=\sup _{i} \alpha_{i} P_{V}\left(f\left(x_{i}\right)-g\left(x_{i}\right)\right) \leq \epsilon$, as we desired.

\section{3. $\mathcal{H}^{\infty}(\mathcal{U} ; \boldsymbol{F})$ and the Approximation Property}

We say that a locally convex space $E$ has the approximation property (AP for short) if for every convex compact and balanced subset $K$ of $E$ and every neighborhood of zero $V$ in $E$ there is a finite rank mapping $T \in L(E ; E)$ such that $T x-x \in V$ for all $x \in K$. It is well known that each complemented subspace of a locally convex space with the AP has the AP.

By [26, Proposition 5.2] we have the following: 
Remark 3.1. Let $E$ and $F$ be Banach spaces, let $U$ be an open subset of $E$, and let $\mathcal{U}=\left(U_{n}\right)_{n \in \mathbb{N}}$ be an increasing countable open cover of $U$ such that every $U_{n}$ is bounded and balanced. If $\left(\sigma_{m} f\right)_{m \in \mathbb{N}}$ denotes the sequence of Cesàro means defined in Proposition 5.2 of [26], then one can readily prove that $\sigma_{m} f \longrightarrow f$ in $\left(\mathcal{H}^{\infty}(\mathcal{U} ; F), \tau_{\gamma}\right)$ for each $f \in \mathcal{H}^{\infty}(\mathcal{U} ; F)$.

In [27], by using a result of K. D. Bierstedt and R. Meise [6, Satz 1.2], J. Mujica proves that a Banach space $E$ has the AP if and only if the complete (LB)-space $G^{\infty}(\mathcal{U})$ has the AP. The following result, whose proof is based on a technique of J. Mujica [26, Theorem 5.4], improves this result by presenting new equivalences. The symbol $\Lambda$ denotes a directed set.

Theorem 3.2. Let $E$ be a Banach space, let $U$ be an open balanced subset of $E$, and let $\mathcal{U}=\left(U_{n}\right)_{n \in \mathbb{N}}$ be a sequence of bounded, balanced, open subsets of $U$ such that $U=\bigcup_{n=1}^{\infty} U_{n}$ and $\rho_{n} U_{n} \subset U_{n+1}$, with $\rho_{n}>1$, for every $n \in \mathbb{N}$. The following are equivalent:

(i) E has the AP.

(ii) $\mathcal{H}^{\infty}(\mathcal{U} ; F)={\overline{\mathcal{P}_{f}(E ; F)}}^{\tau_{\gamma}}$ for every Banach space $F$ (equivalently for every locally convex space $F$ ).

(iii) $\mathcal{H}^{\infty}(\mathcal{U} ; F)=\overline{\mathcal{H}} \infty(\mathcal{U}) \otimes F^{\tau_{\gamma}}$ for every Banach space $F$ (equivalently for every locally convex space $F$ ).

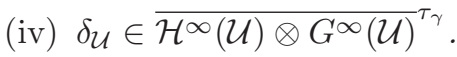

(v) $G^{\infty}(\mathcal{U})$ has the $A P$.

(vi) For every Banach space $F$, and for every open, balanced subset $V \subset F$, $\mathcal{H}^{\infty}(\mathcal{V} ; E)=\overline{\mathcal{H}}^{\infty}(\mathcal{V}) \otimes E^{\tau}$, where $\mathcal{V}=\left(V_{n}\right)_{n \in \mathbb{N}}$ is a sequence of bounded, balanced, open subsets of $V$ such that $V=\bigcup_{n=1}^{\infty} V_{n}$ and $\rho_{n} V_{n} \subset V_{n+1}$, with $\rho_{n}>1$, for every $n \in \mathbb{N}$.

(vii) $I_{U} \in \overline{\mathcal{H}} \infty(\mathcal{U}) \otimes E^{\tau_{\gamma}}$.

Proof. (i) $\Longrightarrow$ (ii): Let $F$ be a Banach space, let $f \in \mathcal{H}^{\infty}(\mathcal{U} ; F)$ and let $p$ be a continuous seminorm on $\left(\mathcal{H}^{\infty}(\mathcal{U} ; F), \tau_{\gamma}\right)$. Then by Remark 3.1 there is a $P \in \mathcal{P}(E ; F)$ such that $p(P-f)<\frac{\epsilon}{2}$. Let $P=P^{0}+P^{1}+\ldots+P^{n}$, where $P^{j} \in \mathcal{P}\left({ }^{j} E ; F\right)$ for every $j=0,1, \ldots, n$. By Proposition $2.2 \tau_{\gamma}=\tau_{c}$ on $\mathcal{P}\left({ }^{k} E ; F\right)$ for every $k \in \mathbb{N}_{0}$. Hence, by [26, Lemma 5.3] there are $Q^{j} \in \mathcal{P}_{f}\left({ }^{j} E ; F\right)$ such that $p\left(Q^{j}-P^{j}\right)<\frac{\epsilon}{2(n+1)}$ for every $j=0,1, \ldots, n$. Note that $Q^{0}+\cdots+Q^{n}=Q \in \mathcal{P}_{f}(E ; F)$, and since $p(Q-P) \leq p\left(Q^{0}-P^{0}\right)+\cdots+p\left(Q^{n}-P^{n}\right)<\frac{\epsilon}{2}$, then $p(Q-f)<\epsilon$. Hence we have (ii). 
(ii) $\Longrightarrow$ (iii): Since $\mathcal{P}_{f}(E ; F) \subset \mathcal{H}^{\infty}(\mathcal{U}) \otimes F \subset \mathcal{H}^{\infty}(\mathcal{U} ; F)$, and $F$ is a Banach space,

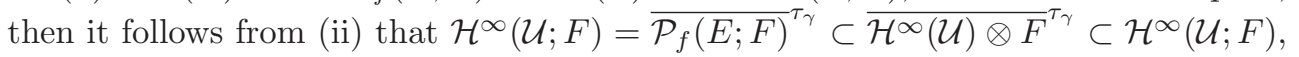
and we have (iii).

The fact that each of the conditions (ii) or (iii) for every Banach space $F$ is equivalent to the corresponding condition for every locally convex space $F$ follows from Proposition 2.8.

(iii) $\Longrightarrow$ (iv): By (iii) and by Proposition 2.8 , we get

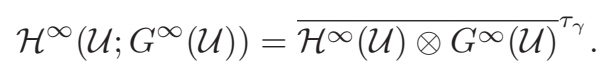

Since by Theorem $2.3 \delta_{\mathcal{U}} \in \mathcal{H}^{\infty}\left(\mathcal{U} ; G^{\infty}(\mathcal{U})\right)$ we have (iv).

(iv) $\Longrightarrow$ (v): From (iv) there is a net $\left(g_{\alpha}\right)_{\alpha \in \Lambda} \subset \mathcal{H}^{\infty}(\mathcal{U}) \otimes G^{\infty}(\mathcal{U})$ such that $g_{\alpha} \stackrel{\tau_{\gamma}}{\longrightarrow}$ $\delta_{\mathcal{U}}$. By Proposition 2.7 we have a corresponding net $\left(T_{g_{\alpha}}\right)_{\alpha \in \Lambda} \subset G^{\infty}(\mathcal{U})^{\prime} \otimes G^{\infty}(\mathcal{U})$. By Theorem 2.3 the identity mapping $I: G^{\infty}(\mathcal{U}) \longrightarrow G^{\infty}(\mathcal{U})$ corresponds to $\delta_{\mathcal{U}}$. Since by Proposition $\left.2.5\left(\mathcal{H}^{\infty}\left(\mathcal{U} ; G^{\infty}(\mathcal{U})\right), \tau_{\gamma}\right) \simeq L\left(G^{\infty}(\mathcal{U}) ; G^{\infty}(\mathcal{U})\right), \tau_{c}\right)$ then we have that $T_{g_{\alpha}} \stackrel{\tau_{c}}{\longrightarrow} I$, i.e., $I \in \bar{G}^{\infty}(\mathcal{U})^{\prime} \otimes G^{\infty}(\mathcal{U})^{\tau_{c}}$. Hence $G^{\infty}(\mathcal{U})$ has the AP.

(v) $\Longrightarrow$ (i): Since, by [27, Proposition 2.4], $E$ is topologically isomorphic to a complemented subspace of $G^{\infty}(\mathcal{U})$, then by (v) $E$ has the AP.

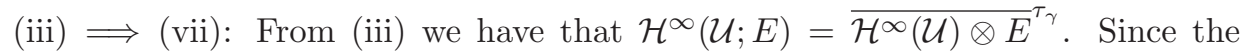
identity mapping $I: U \longrightarrow U \subset E$ is bounded on each $U_{n}$, then $I \in \mathcal{H}^{\infty}(\mathcal{U} ; E)$, and we have (vii).

(vii) $\Longrightarrow$ (i): Suppose that $I \in \overline{\mathcal{H}}^{\infty}(\mathcal{U}) \otimes E^{\tau_{\gamma}}$, where $I$ is the identity mapping on $U$. We will show that $\left.I\right|_{U_{n}} \in \overline{\mathcal{H}}^{\infty}\left(U_{n}\right) \otimes E^{\tau_{\gamma}}$ for each $n \in \mathbb{N}$. By hypotheses there is a net $\left(g_{\alpha}\right)_{\alpha \in \Lambda} \subset \mathcal{H}^{\infty}(\mathcal{U}) \otimes E$ such that $g_{\alpha} \stackrel{\tau_{\gamma}}{\longrightarrow} I$. By Proposition 2.6 for each $\alpha \in \Lambda, g_{\alpha}$ corresponds to a sequence $\left(g_{\alpha}^{n}\right)_{n \in \mathbb{N}}$ in $\operatorname{proj}_{n \in \mathbb{N}}\left[\mathcal{H}^{\infty}\left(U_{n}\right) \otimes E\right]$, where $g_{\alpha}^{n}=\left.g_{\alpha}\right|_{U_{n}} \in \mathcal{H}^{\infty}\left(U_{n}\right) \otimes E$ for every $n \in \mathbb{N}$. Hence we have $\left.\left.g_{\alpha}\right|_{U_{n}} \stackrel{\tau_{\gamma}}{\longrightarrow} I\right|_{U_{n}}$ for each $n \in \mathbb{N}$. Then $\left.I\right|_{U_{n}} \in \overline{\mathcal{H}}^{\infty}\left(U_{n}\right) \otimes E^{\tau_{\gamma}}$, and by [26, Theorem 5.4] $E$ has the AP.

(i) $\Longrightarrow$ (vi): Let $V$ be an open, balanced subset of $F$, and let $\mathcal{V}=\left(V_{n}\right)_{n \in \mathbb{N}}$ be a sequence of balanced, bounded, open subsets of $V$ such that $V=\bigcup_{n=1}^{\infty} V_{n}$ and $\rho_{n} V_{n} \subset V_{n+1}$, with $\rho_{n}>1$, for every $n \in \mathbb{N}$. Then by (i) we have that $\left(L\left(G^{\infty}(\mathcal{V}) ; E\right), \tau_{c}\right)=\overline{G^{\infty}(\mathcal{V})^{\prime} \otimes E}$. (See [16, §5, Proposition 35], or [20, §43, 1. (1)].) Hence by Propositions 2.5 and 2.7 we have that $\left(\mathcal{H}^{\infty}(\mathcal{V} ; E), \tau_{\gamma}\right)=\overline{\mathcal{H}^{\infty}(\mathcal{V}) \otimes E}$.

(vi) $\Longrightarrow$ (i): From (vi) we have that $\left(\mathcal{H}^{\infty}(\mathcal{V} ; E), \tau_{\gamma}\right)=\overline{\mathcal{H}^{\infty}(\mathcal{V}) \otimes E}$. Thus by Propositions 2.5 and 2.7 we have that $\left(L\left(G^{\infty}(\mathcal{V}) ; E\right), \tau_{c}\right)=\overline{G^{\infty}(\mathcal{V})^{\prime} \otimes E}$. We will prove that $F^{\prime} \otimes E$ is $\tau_{c}$-dense in $L(F ; E)$ for each Banach space $F$. Let $A \in$ $L(F ; E)$. Then by $\left[27\right.$, Proposition 2.4] there are mappings $S \in L\left(F ; G^{\infty}(\mathcal{V})\right)$ and $R \in L\left(G^{\infty}(\mathcal{V}) ; F\right)$ such that $R \circ S(y)=y$ for all $y \in F$. Then $A \circ R \in L\left(G^{\infty}(\mathcal{V}) ; E\right)$ and hence there is net $\left(B_{\alpha}\right)_{\alpha \in \Lambda} \subset G^{\infty}(\mathcal{V})^{\prime} \otimes E$ such that $B_{\alpha} \stackrel{\tau_{c}}{\longrightarrow} A \circ R$ in $\left(L\left(G^{\infty}(\mathcal{V}) ; E\right), \tau_{c}\right)$. Thus, $\left(B_{\alpha} \circ S\right)_{\alpha \in \Lambda} \subset F^{\prime} \otimes E$ and $B_{\alpha} \circ S \stackrel{\tau_{c}}{\longrightarrow} A \circ R \circ S=A$ in $\left(L(F ; E), \tau_{c}\right)$. Therefore we showed that $L(F ; E)={\overline{F^{\prime} \otimes E^{\tau_{c}}}}^{\tau}$. Then by [21, Theorem 1.e.4] $E$ has the AP. 
From the previous theorem and by [27, Proposition 7.1], we obtain the following result for the holomorphic mappings of bounded type.

Corollary 3.3. Let $E$ be a Banach space, let $U$ be an open balanced subset of $E$. The following are equivalent:

(i) E has the AP.

(ii) $\mathcal{H}_{b}(U ; F)=\overline{\mathcal{P}}(E ; F)^{\tau_{\gamma}}$ for every Banach space $F$ (equivalently for every locally convex space $F$ ).

(iii) $\mathcal{H}_{b}(U ; F)=\overline{\mathcal{H}}_{b}(U) \otimes F^{\tau_{\gamma}}$ for every Banach space $F$ (equivalently for every locally convex space $F$ ).

(iv) $\delta_{\mathcal{U}} \in{\overline{\mathcal{H}_{b}(U) \otimes G_{b}(U)}}^{\tau_{\gamma}}$.

(v) $G_{b}(U)$ has the $A P$.

(vi) For every Banach space $F$, and for every open, balanced subset $V \subset F$, $\mathcal{H}_{b}(V ; E)=\overline{\mathcal{H}}_{b}(V) \otimes E^{\tau_{\gamma}}$.

(vii) $I_{U} \in \overline{\mathcal{H}}_{b}(U) \otimes E^{\tau_{\gamma}}$.

\section{The Compact Approximation Property in locally convex spa- ces}

Let $(L(E ; F), c)$ denote the vector space $L(E ; F)$, endowed with the topology of uniform convergence on all compact, convex, balanced subsets of $E$, i.e, the topology which admits as the base of neighborhoods of zero the sets of the form $W(K ; V)=$ $\{T \in L(E ; F): T(K) \subset V\}$, where $K$ varies over compact convex and balanced subsets of $E$, and $V$ varies over neighborhoods of zero of $F$, which are convex, balanced and closed. In case $F=\mathbb{C}$ we will denote $(L(E ; F), c)$ by $E_{c}^{\prime}$.

We denote by $A^{\circ}$ the polar of a set $A \subset E$. Let $L_{\epsilon}\left(E_{c}^{\prime} ; F\right)$ denote the vector space $L\left(E_{c}^{\prime} ; F\right)$, endowed with the topology of uniform convergence on the equicontinuous subsets of $E^{\prime}$, i.e, the topology which admits as the base of neighborhoods of zero the sets of the form $W\left(U^{\circ} ; V\right)=\left\{T \in L\left(E_{c}^{\prime} ; F\right): T\left(U^{\circ}\right) \subset V\right\}$, where $U$ and $V$ varies over neighborhoods of zero of $E$ and $F$ respectively, which are convex, balanced and closed.

The following proposition is well known, and is easy to prove anyway.

Proposition 4.1. Let $E$ and $F$ be locally convex spaces.

(i) The inclusion

$$
J:\left(L((E ; F), c) \hookrightarrow L_{\epsilon}\left(\left(E_{c}^{\prime}\right)_{c}^{\prime} ; F\right)\right.
$$

is always an imbedding. If $\left(E_{c}^{\prime}\right)_{c}^{\prime}=E$, then $J$ is surjective. 
(ii) The identity

$$
I:\left(L\left(E_{c}^{\prime} ; F\right), c\right) \longrightarrow L_{\epsilon}\left(E_{c}^{\prime} ; F\right)
$$

is always continuous. If $\left(E_{c}^{\prime}\right)_{c}^{\prime}=E$, then $I$ is a homeomorphism.

In the preceding proposition $\left(E_{c}^{\prime}\right)_{c}^{\prime}=E$ holds, in particular, if $E$ is a Mackey space, or, if $E=F_{c}^{\prime}$ with $F$ a Mackey space.

For locally convex spaces $E$ and $F$ the $\varepsilon$-product of $E$ and $F$ was introduced by L. Schwartz as the locally convex space $E \epsilon F:=L_{\epsilon}\left(E_{c}^{\prime} ; F\right)$; see L. Schwartz [31,32]. Variants of the $\varepsilon$-product $E \epsilon F$ have been considered by other authors; see K. D. Bierstedt [4], H. Jarchow [18, p. 344] and G. Köthe [20, p. 242]. All these variants coincide when $E$ is complete.

Proposition 4.2 ([31, Exposé no. 8]). Let $E$ and $F$ be locally convex spaces. Then $F \varepsilon E$ is topologically isomorphic to $E \varepsilon F$.

Proposition 4.3. Let $E$ and $F$ be locally convex spaces. Then $T \in L\left(F_{c}^{\prime} ; E\right)$ is compact if and only if the adjoint operator $T^{\prime} \in L\left(E_{c}^{\prime} ; F\right)$ is compact.

A locally convex space $E$ is said to have the compact approximation property (CAP for short) if for every convex, compact and balanced subset $K$ of $E$ and every neighborhood of zero $V$ in $E$ there is a compact mapping $T \in L(E ; E)$ such that $T x-x \in V$ for all $x \in K$. If a locally convex space $E$ has the CAP, then each complemented subspace of $E$ has it. The proof of the following proposition follows from an argument in $[20, \S 43,4 .(2)$ and $(3)]$.

Proposition 4.4. (i) The locally convex direct sum $E=\bigoplus_{\alpha \in \Lambda} E_{\alpha}$ of locally convex spaces $E_{\alpha}$ has the CAP if and only if each $E_{\alpha}$ has the CAP.

(ii) The topological product $E=\prod_{\alpha \in \Lambda} E_{\alpha}$ of locally convex spaces $E_{\alpha}$ has the CAP if and only if each $E_{\alpha}$ has the CAP.

The following result is similar to the characterization of the AP due to A. Grothendieck [16, Chapitre I, no. 5] and L. Schwartz [31, Exposé no. 14].

Proposition 4.5. Let $E$ be a locally convex space. Let us consider the following conditions:

(i) E has the CAP.

(ii) $(L(E ; E), c)=\overline{L_{k}(E ; E)}$.

(iii) $(L(E ; F), c)=\overline{L_{k}(E ; F)}$ for every locally convex space $F$.

(iv) $(L(F ; E), c)=\overline{L_{k}(F ; E)}$ for every locally convex space $F$.

(v) $F \epsilon E=\overline{L_{k}\left(F_{c}^{\prime} ; E\right)}$ for every locally convex space $F$. 
(vi) $E \epsilon F=\overline{L_{k}\left(E_{c}^{\prime} ; F\right)}$ for every locally convex space $F$.

Always we have

$$
\text { (i) } \Longleftrightarrow \text { (ii) } \Longleftrightarrow \text { (iii) } \Longleftrightarrow \text { (iv) } \Longrightarrow \text { (v) } \Longleftrightarrow \text { (vi), }
$$

and moreover (v) implies

(iv') $(L(F ; E), c)=\overline{L_{k}(F ; E)}$ for every locally convex space $F$ such that $\left(F_{c}^{\prime}\right)_{c}^{\prime}=F$.

Proof. Let $F$ be a locally convex space. If $E$ has the CAP, then there is a net $\left(T_{\alpha}\right)_{\alpha \in \Lambda} \subset L_{k}(E ; E)$ such that $T_{\alpha} \stackrel{c}{\longrightarrow} I_{E}$. Hence for any $T \in L(E ; F)$ we have that $T \circ T_{\alpha} \stackrel{c}{\longrightarrow} T$, which shows that (i) implies (iii), and for any $T \in L(F ; E)$ we have that $T_{\alpha} \circ T \stackrel{c}{\longrightarrow} T$, which shows that (i) implies (iv). While the equivalence of (v) and (vi) is a consequence of Propositions 4.2 and 4.3, it follows from (iv) that $\left(L\left(F_{c}^{\prime} ; E\right), c\right)=$ $\overline{L_{k}\left(F_{c}{ }^{\prime} ; E\right)}$ for every locally convex space $F$, and hence, by Proposition 4.1 (ii) we have (v). If conversely $(\mathrm{v})$ holds we have that $L_{\epsilon}\left(\left(F_{c}^{\prime}\right)_{c}^{\prime} ; E\right)=\overline{L_{k}\left(\left(F_{c}^{\prime}\right)_{c}^{\prime} ; E\right)}$ for every locally convex space $F$, and if $\left(F_{c}^{\prime}\right)_{c}^{\prime}=F$, then by Proposition 4.1 (i) we have (iv'). Since the another implications are trivial, the proof is complete.

We do not know in the previous proposition if in general (v) implies (iv), and we do not know if each of the conditions (iii), (iv), (v) or (vi) for every Banach space $F$ is equivalent to the corresponding condition for every locally convex space $F$.

Corollary 4.6. Let $E$ be a locally convex space such that $\left(E_{c}^{\prime}\right)_{c}^{\prime}=E$. Then $E$ has the $C A P$ if and only if $E_{c}^{\prime}$ has the CAP. In particular a reflexive locally convex space $E$ has the $C A P$ if and only if $E_{c}^{\prime}$ has the $C A P$.

In [10] C. Boyd, S. Dineen and P. Rueda have proved that if $\left\{E_{n}\right\}_{n=0}^{\infty}$ is an $\mathcal{S}$ absolute decomposition of a locally convex space $E$ (for the definition see [12, §3.3]), then $E$ has the AP if and only if each $E_{n}$ has the AP [10, Proposition 1]. The same proof works also in the case of the CAP.

Proposition 4.7. If $\left\{E_{n}\right\}_{n=0}^{\infty}$ is an $\mathcal{S}$-absolute decomposition of a locally convex space $E$, then $E$ has the CAP if and only if each $E_{n}$ has the CAP.

If $E=\operatorname{proj}_{\alpha \in \Lambda} E_{\alpha}$ is such that each locally convex space $E_{\alpha}$ has the AP, then $E$ has the AP. (This follows from the arguments of the proofs of two results of the book of G. Köthe $[20, \S 43,1,(2)$ and (3)]). But we do not know if the same is true in the case of the CAP, i.e., if $E=\operatorname{proj}_{\alpha \in \Lambda} E_{\alpha}$ is such that each locally convex space $E_{\alpha}$ has the CAP, does $E$ have the CAP?

\section{5. $\mathcal{H}^{\infty}(\mathcal{U} ; \boldsymbol{F})$ and the Compact Approximation Property}

We say that the polynomial $P \in \mathcal{P}(E ; F)$ is compact if every $x \in E$ has a neighborhood $V_{x} \subset E$ such that $P\left(V_{x}\right)$ is relatively compact in $F$. If $E$ and $F$ are Banach 
spaces, we conclude from [3, Proposition 3.4] that a polynomial $P \in \mathcal{P}(E ; F)$ is compact if and only if $P(B)$ is relatively compact in $F$ for every bounded set $B$ in $E$. We denote by $\mathcal{P}_{k}(E ; F)$ the subspace of all compact polynomials in $\mathcal{P}(E ; F)$, and by $\mathcal{P}_{k}\left({ }^{m} E ; F\right)$ the subspace of all polynomials in $\mathcal{P}\left({ }^{m} E ; F\right)$ which are compact, for every $m \in \mathbb{N}_{0}$.

A function $f: E \longrightarrow F$ is called weakly continuous on the bounded sets of $E$ if for each bounded set $B \subset E, \epsilon>0, x_{0} \in B$, and each continuous seminorm $p$ on $F$ there are $\varphi_{1}, \ldots, \varphi_{k} \in E^{\prime}$ and $\delta>0$ such that if $x \in B,\left|\varphi_{i}\left(x_{0}-x\right)\right|<\delta,(i=1, \ldots, k)$, then $p\left(f\left(x_{0}\right)-f(x)\right)<\epsilon$.

A function $f: E \longrightarrow F$ is called weakly uniformly continuous in the bounded sets of $E$ if for each bounded set $B \subset E, \epsilon>0$, and each continuous seminorm $p$ on $F$ there are $\varphi_{1}, \ldots, \varphi_{k} \in E^{\prime}$ and $\delta>0$ such that if $x, y \in B,\left|\varphi_{i}(x-y)\right|<\delta$, $(i=1, \ldots, k)$, then $p(f(x)-f(y))<\epsilon$.

Let $\mathcal{P}_{w}(E ; F)$ (resp. $\left.\mathcal{P}_{w u}(E ; F)\right)$ denote the subspace of all members of $\mathcal{P}(E ; F)$ which are weakly (resp. weakly uniformly) continuous in the bounded sets of $E$ and let $\mathcal{P}_{w}\left({ }^{m} E ; F\right)$ (resp. $\left.\mathcal{P}_{w u}\left({ }^{m} E ; F\right)\right)$ denote the subspace of all members of $\mathcal{P}\left({ }^{m} E ; F\right)$ which are weakly (resp. weakly uniformly) continuous in the bounded sets of $E$, for every $m \in \mathbb{N}_{0}$. We refer to [1] and [2] for the properties of $\mathcal{P}_{w}(E ; F)$ and $\mathcal{P}_{w u}(E ; F)$ on infinite dimensional spaces.

If $E$ is a Banach space, for each $m \in \mathbb{N}$, we let $Q\left({ }^{m} E\right)$ be the space of all linear forms on $\left.\mathcal{P}{ }^{m} E\right)$ which when restricted to each locally bounded set are $\tau_{c}$-continuous.

In [8] C. Boyd gave an $\mathcal{S}$-absolute decomposition of $G(U)$, the predual of $\mathcal{H}(U), U$ an open balanced subset of a locally convex space $E$. By a modification of the proof of $\left[8\right.$, Proposition 4], it is possible to prove a similar result for $G^{\infty}(\mathcal{U})$.

Proposition 5.1. Let $U$ be an open, balanced subset of a Banach space E, and let $\mathcal{U}=\left(U_{n}\right)_{n \in \mathbb{N}}$ be an increasing countable open cover of bounded, balanced, open subsets of $U$. Then $\left\{Q\left({ }^{n} E\right)\right\}_{n=0}^{\infty}$ is an $\mathcal{S}$-absolute decomposition for $G^{\infty}(\mathcal{U})$.

Now we give the main result of this section which asserts that a Banach space $E$ has the CAP if and only if the complete (LB)-space $G^{\infty}(\mathcal{U})$ has the CAP. To obtain this result, in the place of the technique of the proof of Theorem 3.2 (whose application does not seem to be possible), we have used the $\mathcal{S}$-absolute decomposition of $G^{\infty}(\mathcal{U})$.

Theorem 5.2. Let $E$ be a Banach space, let $U$ be an open balanced subset of $E$, and let $\mathcal{U}=\left(U_{n}\right)_{n \in \mathbb{N}}$ be a sequence of bounded, balanced, open subsets of $U$ such that $U=\bigcup_{n=1}^{\infty} U_{n}$ and $\rho_{n} U_{n} \subset U_{n+1}$, with $\rho_{n}>1$, for every $n \in \mathbb{N}$. The following are equivalent:

(i) E has the CAP.

(ii) $\mathcal{H}^{\infty}(\mathcal{U} ; F)=\overline{\mathcal{P}},(E ; F)^{\tau_{\gamma}}$ for every Banach space $F$ (equivalently for every complete locally convex space $F$ ). 
(iii) $\mathcal{H}^{\infty}(\mathcal{U} ; F)={\overline{\mathcal{P}_{k}(E ; F)}}^{\tau_{\gamma}}$ for every Banach space $F$ (equivalently for every complete locally convex space $F$ ).

(iv) $\mathcal{H}^{\infty}(\mathcal{U} ; F)={\overline{\mathcal{H}_{K}^{\infty}(\mathcal{U} ; F)}}^{\tau_{\gamma}}$ for every Banach space $F$ (equivalently for every complete locally convex space $F$ ).

(v) $\delta_{\mathcal{U}} \in{\overline{\mathcal{H}_{K}^{\infty}\left(\mathcal{U} ; G^{\infty}(\mathcal{U})\right)}}^{\tau_{\gamma}}$.

(vi) $G^{\infty}(\mathcal{U})$ has the $C A P$.

(vii) For every Banach space $F$, and for every open, balanced subset $V \subset F$, $\mathcal{H}^{\infty}(\mathcal{V} ; E)=\overline{\mathcal{H}}_{K}^{\infty}(\mathcal{V} ; E){ }^{\tau_{\gamma}}$, where $\mathcal{V}=\left(V_{n}\right)_{n \in \mathbb{N}}$ is a sequence of bounded, balanced, open subsets of $V$ such that $V=\bigcup_{n=1}^{\infty} V_{n}$ and $\rho_{n} V_{n} \subset V_{n+1}$, with $\rho_{n}>1$, for every $n \in \mathbb{N}$.

(viii) $I_{U} \in{\overline{\mathcal{H}_{K}^{\infty}(\mathcal{U} ; E)}}^{\tau_{\gamma}}$.

Proof. Firstly we will show the implications for Banach space $F$.

$(\mathrm{i}) \Longrightarrow($ ii): In the proof of implication $(a) \Longrightarrow(b)$ of [11, Theorem 5] using Remark 3.1 and Proposition 2.2 we obtain (ii).

(ii) $\Longrightarrow$ (iii): Let $F$ be a Banach space. It follows from [1, Theorem 2.9] and $\left[2\right.$, Lemma 2.2] that $\mathcal{P}_{w}(E ; F)=\mathcal{P}_{w u}(E ; F) \subset \mathcal{P}_{k}(E ; F) \subset \mathcal{H}^{\infty}(\mathcal{U} ; F)$. Then from (ii) we get (iii).

(iii) $\Longrightarrow\left(\right.$ iv): Since $\mathcal{P}_{k}(E ; F) \subset \mathcal{H}_{K}^{\infty}(\mathcal{U} ; F) \subset \mathcal{H}^{\infty}(\mathcal{U} ; F)$, for $F$ Banach, by (iii) we have (iv).

(iv) $\Longrightarrow$ (viii): It follows from the argument in the implication (iii) $\Longrightarrow$ (vii) of Theorem 3.2 .

(viii) $\Longrightarrow$ (i): By the proof of the implication (vii) $\Longrightarrow$ (i) in Theorem 3.2, we get $\left.I\right|_{U_{n}} \in \overline{\mathcal{H}}_{K}^{\infty}\left(U_{n} ; E\right)^{\tau_{\gamma}}$ for each $n \in \mathbb{N}$. Thus by [11, Theorem 5] $E$ has the CAP.

$(\mathrm{i}) \Longleftrightarrow\left(\right.$ vi): By [11, Corollary 7] $E$ has the CAP if and only if $Q\left({ }^{n} E\right)$ has the CAP for every $n \in \mathbb{N}$. Therefore by Propositions 4.7 and 5.1 for every $n \in \mathbb{N}, Q\left({ }^{n} E\right)$ has the CAP if and only if $G^{\infty}(\mathcal{U})$ has the CAP.

(i) $\Longrightarrow$ (vii): It follows from Propositions 2.5 and 2.7 .

(vii) $\Longrightarrow(\mathrm{i})$ : From (vii) we have that $\left(\mathcal{H}^{\infty}(\mathcal{V} ; E), \tau_{\gamma}\right)=\overline{\mathcal{H}_{K}^{\infty}(\mathcal{V} ; E)}$. Thus by Propositions 2.5 and 2.7 we have that $\left(L\left(G^{\infty}(\mathcal{V}) ; E\right), \tau_{c}\right)=\overline{L_{k}\left(G^{\infty}(\mathcal{V}) ; E\right)}$. From this point on the proof is similar to the proof of the implication (vi) $\Longrightarrow$ (i) in Theorem 3.2.

Now we show that the condition (ii) for every Banach space $F$ is equivalent to the same condition for every complete locally convex space $F$, and the same proof will also work for (iii). Since $\mathcal{P}_{k}(E ; F) \subset \mathcal{H}_{K}^{\infty}(\mathcal{U} ; F)$, as $F$ locally convex space, then by (iii) the same equivalence follows for (iv).

Suppose that $\mathcal{H}^{\infty}(\mathcal{U} ; F)=\overline{\mathcal{P}} w(E ; F)^{\tau}$ for every Banach space $F$. Let $f \in$ $\mathcal{H}^{\infty}(\mathcal{U} ; F)$, as $F$ complete locally convex space, let $\epsilon>0$ and let $\tilde{p}$ be a continuous seminorm on $\left(\mathcal{H}^{\infty}(\mathcal{U} ; F), \tau_{\gamma}\right)$. We may assume that

$$
\tilde{p}(f)=\sup _{j} \alpha_{j} p\left(f\left(x_{j}\right)\right) \text { for all } f \in \mathcal{H}^{\infty}(\mathcal{U} ; F),
$$


where $\left(x_{j}\right)_{j \in \mathbb{N}} \subset U_{m}$ for some $m \in \mathbb{N},\left(\alpha_{j}\right)_{j \in \mathbb{N}}$ is a sequence of positive numbers tending to zero, and $p$ is a continuous seminorm on $F$. The subset $U_{m}$ is bounded, then $B=\bar{\Gamma}\left(f\left(U_{m}\right)\right)$ is bounded in $F$. Since $F$ is a complete locally convex space, the vector subspace generated by $B$, i.e., $F_{B}=\bigcup_{n=1}^{\infty} n B$, is a Banach space, normed by the Minkowski functional $p_{B}$, such that the injection $i_{B}: F_{B} \hookrightarrow F$ is continuous. Since, by hypotheses and the previous equivalences, $E$ has the CAP, then by [11, Theorem 5] we have that $\mathcal{H}^{\infty}\left(U_{m} ; F_{B}\right)=\overline{\mathcal{P}}_{w}\left(E ; F_{B}\right)^{\tau_{\gamma}}$. As $\left.f\right|_{U_{m}} \in \mathcal{H}^{\infty}\left(U_{m} ; F_{B}\right)$ then there is a $P \in \mathcal{P}_{w}\left(E ; F_{B}\right)$ such that

$$
\sup _{j} \alpha_{j} p_{B}\left(P\left(x_{j}\right)-\left.f\right|_{U_{m}}\left(x_{j}\right)\right)<\frac{\epsilon}{c},
$$

where $c>0$ is such that $p(y) \leq c \cdot p_{B}(y)$ for all $y \in F_{B}$. Hence we have that

$$
\begin{aligned}
\tilde{p}(P-f) & =\sup _{j} \alpha_{j} p\left(P\left(x_{j}\right)-f\left(x_{j}\right)\right) \\
& =\sup _{j} \alpha_{j} p\left(P\left(x_{j}\right)-\left.f\right|_{U_{m}}\left(x_{j}\right)\right) \\
& \leq \sup _{j} \alpha_{j} c p_{B}\left(P\left(x_{j}\right)-\left.f\right|_{U_{m}}\left(x_{j}\right)\right) \leq \epsilon .
\end{aligned}
$$

Therefore, since $P=i_{B} \circ P \in \mathcal{P}_{w}(E ; F)$, we have that $\mathcal{H}^{\infty}(\mathcal{U} ; F)={\overline{\mathcal{P}_{w}(E ; F)}}^{\tau_{\gamma}}$ for every complete locally convex space $F$.

Using the above observation we show the equivalence of (v) with the previous conditions.

(iv) $\Longrightarrow(v)$ : Since $G^{\infty}(\mathcal{U})$ is a complete locally convex space, then by (iv) and by

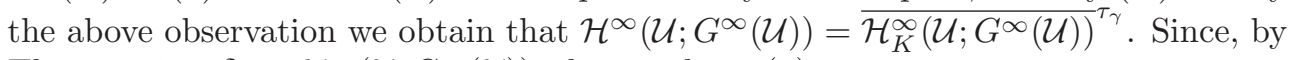
Theorem 2.3, $\delta_{\mathcal{U}} \in \mathcal{H}^{\infty}\left(\mathcal{U} ; G^{\infty}(\mathcal{U})\right)$, then we have (v).

$(\mathrm{v}) \Longrightarrow\left(\right.$ viii): By (v) there is a net $\left(g_{\alpha}\right)_{\alpha \in \Lambda} \subset \mathcal{H}_{K}^{\infty}\left(\mathcal{U} ; G^{\infty}(\mathcal{U})\right)$ such that $g_{\alpha} \stackrel{\tau_{\gamma}}{\longrightarrow} \delta_{\mathcal{U}}$. Let us consider the inclusion $I_{U}: U \longrightarrow U \subset E$. Since $I_{U} \in \mathcal{H}^{\infty}(\mathcal{U} ; E)$ then by Theorem 2.3 there is a $T_{I_{U}} \in L\left(G^{\infty}(\mathcal{U}) ; E\right)$ such that $T_{I_{U}} \circ \delta_{U}=I_{U}$. Therefore we have that $T_{I_{U}} \circ g_{\alpha} \stackrel{\tau_{\gamma}}{\longrightarrow} T_{I_{U}} \circ \delta_{U}=I_{U}$. Since $\left(T_{I_{U}} \circ g_{\alpha}\right)_{\alpha \in \Lambda} \subset \mathcal{H}_{K}^{\infty}(\mathcal{U} ; E)$, we have (viii), and now the proof of the theorem is complete.

We do not know if the method used in Theorem 3.2, to prove that $G^{\infty}(\mathcal{U})$ has the AP when $E$ has it, works in the case of the CAP (because we do not know if $T_{f} \in L_{k}\left(G^{\infty}(\mathcal{U}) ; G^{\infty}(\mathcal{U})\right)$ whenever $\left.f \in \mathcal{H}_{K}^{\infty}\left(\mathcal{U} ; G^{\infty}(\mathcal{U})\right)\right)$. But observe that using the $\mathcal{S}$-absolute decomposition of $G^{\infty}(\mathcal{U})$, it is possible to give another proof that $G^{\infty}(\mathcal{U})$ has the AP when $E$ has it (using [10, Proposition 1], and [26, Corollary 5.5]).

Let $\mathcal{H}_{c}(\mathcal{U} ; F)$ denote the subspace of $\mathcal{H}^{\infty}(\mathcal{U} ; F)$ defined by

$$
\mathcal{H}_{c}(\mathcal{U} ; F)=\{f \in \mathcal{H}(U ; F): f(A) \text { is relatively compact in } F
$$


Note that $\mathcal{H}_{c}(U ; F)=\mathcal{H}_{K}^{\infty}(\mathcal{U} ; F)$ if $\mathcal{U}=\left(U_{n}\right)_{n \in \mathbb{N}}$ is a fundamental sequence of open $U$-bounded sets.

Analogously to the case of the AP (in Corollary 3.3) from Theorem 5.2 in particular we obtain the following result for the holomorphic mappings of bounded type.

Corollary 5.3. Let $E$ be a Banach space, let $U$ be an open balanced subset of $E$. The following are equivalent:

(i) E has the CAP.

(ii) $\mathcal{H}_{b}(U ; F)=\overline{\mathcal{P}}(E ; F)^{\tau_{\gamma}}$ for every Banach space $F$ (equivalently for every complete locally convex space $F$ ).

(iii) $\mathcal{H}_{b}(U ; F)={\overline{\mathcal{P}_{k}(E ; F)}}^{\tau_{\gamma}}$ for every Banach space $F$ (equivalently for every complete locally convex space $F$ ).

(iv) $\mathcal{H}_{b}(U ; F)={\overline{\mathcal{H}_{c}(U ; F)}}^{\tau_{\gamma}}$ for every Banach space $F$ (equivalently for every complete locally convex space $F$ ).

(v) $\delta_{U} \in{\overline{\mathcal{H}}\left(U ; G_{b}(U)\right)^{\tau}}^{\tau_{\gamma}}$.

(vi) $G_{b}(U)$ has the $C A P$.

(vii) For every Banach space $F$, and for every open, balanced subset $V \subset F$, $\mathcal{H}_{b}(V ; E)=\overline{\mathcal{H}}_{c}(V ; E)^{\tau_{\gamma}}$.

(viii) $I_{U} \in{\overline{\mathcal{H}_{c}(U ; E)}}^{\tau_{\gamma}}$.

\section{6. $\mathcal{H}(U ; F)$ and the Compact Approximation Property}

In this section using the $\mathcal{S}$-absolute decomposition of $\mathcal{H}(U ; F)$ and $\mathcal{H}(K ; F)$, due to S. Dineen [12], and $\mathcal{S}$-absolute decomposition of $G(U)$ and $G(K)$, due to C. Boyd [8], we obtain necessary and sufficient conditions for $G(U), U$ balanced open subset of a locally convex space (in particular of a Fréchet space) $E$, to have the CAP.

Let $U$ be an open subset of a locally convex space $E$ and let $F$ be a normed space. A seminorm $p$ on $\mathcal{H}(U ; F)$ is said to be ported by the compact set $K$ of $U$ if, for each neighborhood $V, K \subset V \subset U$, there is $C_{V}>0$ such that $p(f) \leq C_{V}\|f\|_{V}$, for all $f \in \mathcal{H}(U ; F)$. The $\tau_{\omega}$-topology on $\mathcal{H}(U ; F)$ is the topology generated by all seminorms ported by compact subsets of $U$.

Let $U$ be an open subset of a locally convex space $E$ and let $F$ be a normed space. A seminorm $p$ on $E$ is called $\tau_{\delta}$-continuous, if for each countable increasing open cover $\left\{U_{n}\right\}_{n=1}^{\infty}$ of $U$ there are an integer $n_{0}$ and $C>0$ such that $p(f) \leq C\|f\|_{U_{n_{0}}}$, for all $f \in \mathcal{H}(U ; F)$. The $\tau_{\delta}$-topology on $\mathcal{H}(U ; F)$ is the topology generated by all $\tau_{\delta}$-continuous seminorms. 
Let $K$ be a compact subset of a locally convex space $E$ and let $F$ be a locally convex space. We denote by $\mathcal{H}(K ; F)$ the space of holomorphic germs from $K$ into $F$.

From Proposition 4.7, and [12, Propositions 3.36 and 4.29] we obtain the following:

Proposition 6.1. Let $E$ be a locally convex space and let $F$ be a Banach space. The following are equivalent:

(i) $\left(\mathcal{P}\left({ }^{m} E ; F\right), \tau_{c}\right)$ has the $C A P$ for every $m \in \mathbb{N}$.

(ii) $\left(\mathcal{H}(U ; F), \tau_{c}\right)$ has the CAP for one (hence for every) balanced open subset $U$ of $E$.

(iii) $\left(\mathcal{H}(K ; F), \tau_{c}\right)$ has the CAP for one (hence for every) balanced compact subset $K$ of $E$.

Proposition 6.2. Let $E$ be a locally convex space and let $F$ be a Banach space. The following are equivalent:

(i) $\left(\mathcal{P}\left({ }^{m} E ; F\right), \tau_{\omega}\right)$ has the $C A P$ for every $m \in \mathbb{N}$.

(ii) $\left(\mathcal{H}(U ; F), \tau_{\omega}\right)$ has the CAP for one (hence for every) balanced open subset $U$ of $E$.

(iii) $\left(\mathcal{H}(U ; F), \tau_{\delta}\right)$ has the $C A P$ for one (hence for every) balanced open subset $U$ of $E$.

(iv) $\left(\mathcal{H}(K ; F), \tau_{\omega}\right)$ has the CAP for one (hence for every) balanced compact subset $K$ of $E$.

In [22] P. Mazet shows that for an open subset $U$ of a locally convex space $E$ there is a complete locally convex space $G(U)$ and a mapping $\delta_{U} \in \mathcal{H}(U ; G(U))$ with the following universal property: For each complete locally convex space $F$ and each mapping $f \in \mathcal{H}(U ; F)$ there is a unique $T_{f} \in L(G(U) ; F)$ such that $f=T_{f} \circ \delta_{U}$. In particular, taking $F=\mathbb{C}$ we see that $G(U)$ is a predual of $\mathcal{H}(U)$. In [28] J. Mujica and L. Nachbin gave another proof of this result, where $G(U)$ is defined in the following way: If $\mathcal{U}=\left(U_{j}\right)_{j \in \mathbb{N}}$ is a countable open cover of $U$, and $\alpha=\left(\alpha_{j}\right)_{j \in \mathbb{N}}$ is a sequence of strictly positive numbers, then we set $B_{\mathcal{U}}^{\alpha}=\left\{f \in \mathcal{H}(U):\|f\|_{U_{j}} \leq \alpha_{j}\right.$ for every $\left.j\right\}$, and define $G(U)=\left\{u \in \mathcal{H}(U)^{\prime}:\left.u\right|_{B_{\mathcal{U}}^{\alpha}}\right.$ is $\tau_{c}$-continuous for each $\left.\alpha\right\}$, endowed with the topology of uniform convergence on all the sets $B_{\mathcal{U}}^{\alpha}$.

While $\mathcal{H}(U)$ has a predual, the spaces $\mathcal{P}\left({ }^{n} E\right)$, for each $n \in \mathbb{N}$, and $\mathcal{H}(K)$ also have preduals. In [29] R. Ryan shows that for each locally convex space $E$ and each $n \in \mathbb{N}$ there is a polynomial $\delta_{n} \in \mathcal{P}\left({ }^{n} E ; \widehat{\bigotimes}_{s, n, \pi} E\right)$ with the following universal property: Given a complete locally convex space $F$ and $P \in \mathcal{P}\left({ }^{n} E ; F\right)$ there is a unique $T_{P} \in L\left(\widehat{\bigotimes}_{s, n, \pi} E ; F\right)$ such that $P=T_{P} \circ \delta_{n}$, where $\widehat{\bigotimes}_{s, n, \pi} E$ is a completion of n-fold symmetric tensor product of $E$ with itself, endowed with the projective topology or the $\pi$ topology (for the definition see [12, pgs. 18-19]). In [7] C. Boyd adapting the 
proof of [28, Theorem 2.1] gave an alternative proof of this result, where $\widehat{\otimes}_{s, n, \pi} E$ is substituted by $Q\left({ }^{n} E\right)$ which is defined in the following way: Let $\alpha$ be a seminorm on $E$ and let $B_{\alpha}^{n}=\left\{P \in \mathcal{P}\left({ }^{n} E\right):|P(x)| \leq 1\right.$ for $x \in E$ with $\left.\alpha(x) \leq 1\right\}$. Then we define $Q\left({ }^{n} E\right)=\left\{\phi \in \mathcal{P}\left({ }^{n} E\right)^{\prime}:\left.\phi\right|_{B_{\alpha}^{n}}\right.$ is $\tau_{c^{-}}$continuous for each $\left.\alpha\right\}$, endowed with the topology of uniform convergence on all the sets $B_{\alpha}^{n}$. Then $\widehat{\otimes}_{s, n, \pi} E$ is topologically isomorphic to $Q\left({ }^{n} E\right)$ [7, Corollary 2.11], and using this description of $Q\left({ }^{n} E\right)$, C. Boyd [7] (see also [8]) gave an $\mathcal{S}$-absolute decomposition for $G(U)$ with $U$ open balanced subset of $E$. Furthermore he proved the existence of a complete locally convex space $G(K)$, with $K$ compact in $E$, such that $\mathcal{H}(K)=G(K)_{i}^{\prime}$, where $G(K)_{i}^{\prime}$ denotes the inductive dual of $G(K)$, and gave also an $\mathcal{S}$-absolute decomposition for $G(K)$, where $K$ is a compact balanced subset in $E$. We refer to [7], (see also [8]) for the properties of $G(K)$ and to [5] for the definition of the inductive dual of a locally convex space.

From Proposition 4.7, and [8, Propositions 4 and 5] we get immediately the following:

Proposition 6.3. For a locally convex space $E$ the following are equivalent:

(i) $Q\left({ }^{m} E\right)$ has the $C A P$ for every $m \in \mathbb{N}$.

(ii) $G(U)$ has the CAP for one (hence for every) balanced open subset $U$ of $E$.

(iii) $G(K)$ has the CAP for one (hence for every) balanced compact subset $K$ of $E$.

Theorem 6.4 ([30, Theorem 2.3]). Let $U$ be an open subset of an $k$-space $E$ and let $F$ be a quasi-complete locally convex space. Then the mapping

$$
f \in\left(\mathcal{H}(U ; F), \tau_{c}\right) \longrightarrow S_{f} \in L_{\epsilon}\left(F_{c}^{\prime} ;\left(\mathcal{H}(U), \tau_{c}\right)\right)
$$

is a topological isomorphism, where for every $f \in \mathcal{H}(U ; F), S_{f}: F_{c}^{\prime} \longrightarrow\left(\mathcal{H}(U), \tau_{c}\right)$ is defined by $S_{f}(\varphi)=\varphi \circ f$ if $\varphi \in F_{c}^{\prime}$. The inverse mapping is given by

$$
S \in L_{\epsilon}\left(F_{c}^{\prime} ;\left(\mathcal{H}(U), \tau_{c}\right)\right) \longrightarrow S^{\prime} \circ \varepsilon \in\left(\mathcal{H}(U ; F), \tau_{c}\right),
$$

where $S^{\prime}$ is the adjoint mapping of $S$, and $\varepsilon: U \longrightarrow\left(\mathcal{H}(U), \tau_{c}\right)_{c}^{\prime}$ is the evaluation mapping defined by $\varepsilon(x): g \in \mathcal{H}(U) \longrightarrow g(x) \in \mathbb{C}$ for every $x \in U$ and $g \in \mathcal{H}(U)$.

Let $E$ and $F$ be locally convex spaces and let $U$ be an open subset of $E$. Following R. Aron and M. Schottenloher [3] we say that a mapping $f \in \mathcal{H}(U ; F)$ is compact if each $x \in U$ has a neighborhood $V_{x} \subset U$ such that $f\left(V_{x}\right)$ is relatively compact in $F$.

Proposition 6.5. Let $E$ be a metrizable space and let $F$ be a quasi-complete locally convex space.

(i) If $U$ is an open subset of $E$, then $f \in \mathcal{H}(U ; F)$ is compact if and only if $S_{f} \in$ $L\left(F_{c}^{\prime} ;\left(\mathcal{H}(U), \tau_{c}\right)\right)$ is compact. 
(ii) Let $n \in \mathbb{N}$, then $P \in \mathcal{P}\left({ }^{n} E ; F\right)$ is compact if and only if $S_{P} \in L\left(F_{c}^{\prime} ;\left(\mathcal{P}\left({ }^{n} E\right), \tau_{c}\right)\right)$ is compact.

Proof. We will prove only the part (i) since the same proof works for (ii).

$(\Longrightarrow)$ Let us consider the mapping

$$
f \in \mathcal{H}(U ; F) \longrightarrow S_{f} \in L\left(F_{c}^{\prime} ;\left(\mathcal{H}(U), \tau_{c}\right)\right)
$$

Let $f \in \mathcal{H}(U, F)$ be a compact map and let $a \in U$. Then $a$ has a neighborhood $V_{a}$ in $U$ such that $f\left(V_{a}\right)$ is relatively compact in $F$. Let $K=\overline{f\left(V_{a}\right)}$. Then $K^{\circ} \in \mathcal{V}_{0}\left(F_{c}^{\prime}\right)$, and $S_{f}\left(K^{\circ}\right)$ is locally bounded. Therefore, by [12, Lemma 3.25] $S_{f}\left(K^{\circ}\right)$ is relatively compact in $\left(\mathcal{H}(U), \tau_{c}\right)$. Hence $S_{f}$ is a compact mapping.

$(\Longleftarrow)$ Now we consider the inverse mapping

$$
S \in L\left(F_{c}^{\prime} ;\left(\mathcal{H}(U), \tau_{c}\right)\right) \longrightarrow S^{\prime} \circ \varepsilon \in \mathcal{H}(U ; F),
$$

where $S^{\prime}$ is the adjoint mapping of $S$, and $\varepsilon: U \longrightarrow\left(\mathcal{H}(U), \tau_{c}\right)_{c}^{\prime}$ is the evaluation mapping defined in Theorem 6.4. Let $S \in L\left(F_{c}^{\prime} ;\left(\mathcal{H}(U), \tau_{c}\right)\right)$ be compact. Then by Proposition 4.3, the adjoint mapping $S^{\prime} \in L\left(\left(\mathcal{H}(U), \tau_{c}\right)_{c}^{\prime} ; F\right)$ is also compact. Therefore there is a compact set $K \subset\left(\mathcal{H}(U), \tau_{c}\right)$ such that $S^{\prime}\left(K^{\circ}\right)$ is relatively compact in $F$, where $K^{\circ} \in \mathcal{V}_{0}\left(\left(\mathcal{H}(U), \tau_{c}\right)_{c}^{\prime}\right)$. Since $K$ is bounded in $\left(\mathcal{H}(U), \tau_{c}\right)$ the proof of [25, Proposition 9.15] shows that $K$ is locally bounded. Then for each $a \in U$ there is a neighborhood $V_{a}$ in $U$ and $c_{a}>0$ such that $|g(x)| \leq c_{a}$ for all $x \in V_{a}$ and $g \in K$, i.e., $\frac{1}{c_{a}}|\varepsilon(x)(g)|=\left|\frac{1}{c_{a}} \varepsilon(x)(g)\right| \leq 1$ for all $x \in V_{a}$ and $g \in K$. Since $\frac{1}{c_{a}} \varepsilon(x) \in\left(\mathcal{H}(U), \tau_{c}\right)^{\prime}$ for every $x \in V_{a}$, then $\frac{1}{c_{a}} \varepsilon\left(V_{a}\right) \subset K^{\circ}$. Therefore $\overline{\frac{1}{c_{a}} S^{\prime} \circ \varepsilon\left(V_{a}\right)}=\overline{S^{\prime}\left(\frac{1}{c_{a}} \varepsilon\left(V_{a}\right)\right)} \subset \overline{S^{\prime}\left(K^{\circ}\right)}$. Thus $S^{\prime} \circ \varepsilon\left(V_{a}\right)$ is relatively compact in $F$. Then $S^{\prime} \circ \varepsilon$ is a compact mapping.

By combining Propositions 6.1 and 6.3, for Fréchet spaces we obtain the following result, which is similar to a result of C. Boyd in [9].

Proposition 6.6. Let E be a Fréchet space. Then the following are equivalent:

(i) $\left(\mathcal{H}(U), \tau_{c}\right)$ has the CAP for one (hence for every) balanced open subset $U$ of $E$.

(ii) $\left(\mathcal{H}(K), \tau_{c}\right)$ has the CAP for one (hence for every) balanced compact subset $K$ of $E$.

(iii) $\left(\mathcal{P}\left({ }^{m} E\right), \tau_{c}\right)$ has the $C A P$ for every $m \in \mathbb{N}$.

(iv) $Q\left({ }^{m} E\right)$ has the $C A P$ for every $m \in \mathbb{N}$.

(v) $G(U)$ has the CAP for one (hence for every) balanced open subset $U$ of $E$.

(vi) $G(K)$ has the CAP for one (hence for every) balanced compact subset $K$ of $E$.

(vii) E has the CAP. 
(viii) $\left(\mathcal{P}\left({ }^{m} E ; F\right), \tau_{c}\right)=\overline{\mathcal{P}_{k}\left({ }^{m} E ; F\right)}$ for every locally convex space $F$ (equivalently for every Fréchet space $F$ ) and for every $m \in \mathbb{N}$.

Proof. The equivalence of (i) $\Longleftrightarrow$ (ii) $\Longleftrightarrow$ (iii) follows from Proposition 6.1 and the equivalence of (iv) $\Longleftrightarrow(\mathrm{v}) \Longleftrightarrow$ (vi) follows from Proposition 6.3.

(iii) $\Longleftrightarrow\left(\right.$ iv): Since $E$ is a Fréchet space then, for every $m \in \mathbb{N}, Q\left({ }^{m} E\right)$ also is a Fréchet space (see [29, pg. 50]). Thus by Corollary 4.6, $Q\left({ }^{m} E\right)$ has the CAP if and only if $Q\left({ }^{m} E\right)_{c}^{\prime}$ has the CAP, for every $m \in \mathbb{N}$. Hence, by [29, Corollary 2.2], $Q\left({ }^{m} E\right)_{c}^{\prime}$ has the CAP if and only if $\left(\mathcal{P}\left({ }^{m} E\right), \tau_{c}\right)$ has the CAP, for every $m \in \mathbb{N}$. CAP.

(iii) $\Longrightarrow\left(\right.$ vii): Since, by (iii), $E_{c}^{\prime}$ has the CAP, then by Corollary $4.6 E$ has the

(vii) $\Longrightarrow$ (viii): Let $m \in \mathbb{N}$, let $F$ be a locally convex space and let $p$ be a continuous seminorm on $F$. Let $P \in \mathcal{P}\left({ }^{m} E ; F\right)$, let $K$ be a compact subset of $E$ and let $\epsilon>0$. Then there is $\delta>0$ such that $p(P(x)-P(y)) \leq \epsilon$ whenever $x \in K$ and $d(y, x)<\delta$, where $d$ is the metric on $E$. Let $T \in L_{k}(E ; E)$ such that $d(T x, x)<\delta$ for all $x \in K$. Then $p(P(T(x))-P(x)) \leq \epsilon$ for all $x \in K$. Since $P \circ T \in \mathcal{P}_{k}\left({ }^{m} E ; F\right)$ we have (viii).

(viii) $\Longrightarrow$ (iii): Let $m \in \mathbb{N}$. As pointed out in [3] (after Proposition 1.1), we have the topological isomorphism $\left(\mathcal{P}\left({ }^{m} E ; F\right), \tau_{c}\right) \cong L_{\varepsilon}\left(F_{c}^{\prime} ;\left(\mathcal{P}\left({ }^{m} E\right), \tau_{c}\right)\right)$. Hence from this and by Proposition 6.5, and by (viii) we obtain

$$
\begin{aligned}
F \varepsilon\left(\mathcal{P}\left({ }^{m} E\right), \tau_{c}\right) & =\overline{L_{k}\left(F_{c}^{\prime} ;\left(\mathcal{P}\left({ }^{m} E\right), \tau_{c}\right)\right)} \\
& \text { for every quasi-complete locally convex space } F
\end{aligned}
$$

By [29, Proposition 3.3] and [7, Corollary 2.11] we have that $\left(\mathcal{P}\left({ }^{m} E\right), \tau_{c}\right)_{b}^{\prime}=Q\left({ }^{m} E\right)$, where $b$ indicates the topology of uniform convergence on all bounded subsets of $\left(\mathcal{P}\left({ }^{m} E\right), \tau_{c}\right)$. Since $\left(\mathcal{P}\left({ }^{m} E\right), \tau_{c}\right)$ is a semi-Montel space, then we have that $\left(\mathcal{P}\left({ }^{m} E\right), \tau_{c}\right)_{c}^{\prime}=\left(\mathcal{P}\left({ }^{m} E\right), \tau_{c}\right)_{b}^{\prime}=Q\left({ }^{m} E\right)$, which is a Fréchet space. Thus by (1) we have that

$$
\left(\mathcal{P}\left({ }^{m} E\right), \tau_{c}\right)_{c}^{\prime} \varepsilon\left(\mathcal{P}\left({ }^{m} E\right), \tau_{c}\right)=\overline{L_{k}\left(\left(\left(\mathcal{P}\left({ }^{m} E\right), \tau_{c}\right)_{c}^{\prime}\right)_{c}^{\prime} ;\left(\mathcal{P}\left({ }^{m} E\right), \tau_{c}\right)\right)} .
$$

By [29, Corollary 2.2] and [7, Corollary 2.11] we have that $\left(\mathcal{P}\left({ }^{m} E\right), \tau_{c}\right)=Q\left({ }^{m} E\right)_{c}^{\prime}$. Since $\left(\left(\mathcal{P}\left({ }^{m} E\right), \tau_{c}\right)_{c}^{\prime}\right)_{c}^{\prime}=Q\left({ }^{m} E\right)_{c}^{\prime}=\left(\mathcal{P}\left({ }^{m} E\right), \tau_{c}\right)$, by Proposition 4.1 (i) we have that

$$
\left(L\left(\left(\mathcal{P}\left({ }^{m} E\right), \tau_{c}\right) ;\left(\mathcal{P}\left({ }^{m} E\right), \tau_{c}\right)\right), c\right)=\overline{L_{k}\left(\left(\mathcal{P}\left({ }^{m} E\right), \tau_{c}\right) ;\left(\mathcal{P}\left({ }^{m} E\right), \tau_{c}\right)\right)} .
$$

Then by Proposition $4.5\left(\mathcal{P}\left({ }^{m} E\right), \tau_{c}\right)$ has the CAP.

The fact that the condition (viii) for every Fréchet space $F$ is equivalent to the same condition for every locally convex space $F$ follows from the fact that, for $m=1$, $E$ has the CAP by Proposition 4.5 .

Corollary 6.7. Let $E$ be a Fréchet-Montel space such that $G(E)$ is Montel and let $U$ be a balanced open subset of $E$. Then $E$ has the $C A P$ if and only if $\left(\mathcal{H}(U), \tau_{\delta}\right)$ has the $C A P$. 
Acknowledgements. The results in this paper were obtained in my doctoral thesis at the Universidade Estadual de Campinas, Brazil. I would like to thank my supervisor Professor J. Mujica for his assistance and comments.

\section{References}

[1] R. M. Aron, C. Hervés, and M. Valdivia, Weakly continuous mappings on Banach spaces, J. Funct. Anal. 52 (1983), 189-204.

[2] R. M. Aron and J. B. Prolla, Polynomial approximation of differentiable functions on Banach spaces, J. Reine Angew. Math. 313 (1980), 195-216.

[3] R. M. Aron and M. Schottenloher, Compact holomorphic mappings on Banach spaces and the approximation property, J. Functional Analysis 21 (1976), 7-30.

[4] K. D. Bierstedt, Gewichtete Räume stetiger vektorwertiger Funktionen und das injektive Tensorprodukt. I, J. Reine Angew. Math. 259 (1973), 186-210.

[5] __ An introduction to locally convex inductive limits, Functional Analysis and Its Applications (Nice, 1986), ICPAM Lecture Notes, World Sci. Publishing, Singapore, 1988, pp. 35133.

[6] K. D. Bierstedt and R. Meise, Bemerkungen über die Approximations-eigenschaft lokalkonvexer Funktionenräume, Math. Ann. 209 (1974), 99-107.

[7] C. Boyd, Preduals of the space of holomorphic functions on a Fréchet space, Dublin, 1992.

[8] , Distinguished preduals of spaces of holomorphic functions, Rev. Mat. Univ. Complut. Madrid 6 (1993), 221-231.

[9] - Some topological properties of preduals of spaces of holomorphic functions, Proc. Roy. Irish Acad. Sect. A 94 (1994), 167-178.

[10] C. Boyd, S. Dineen, and P. Rueda, Weakly uniformly continuous holomorphic functions and the approximation property, Indag. Math. (N.S.) 12 (2001), 147-156.

[11] E. Çalışkan, Bounded holomorphic mappings and the compact approximation property in Banach spaces, Port. Math. (N.S.) 61 (2004), 25-33.

[12] S. Dineen, Complex analysis on infinite-dimensional spaces, Springer Monographs in Mathematics, Springer-Verlag London Ltd., London, 1999, ISBN 1-85233-158-5.

[13] S. Dineen and J. Mujica, The approximation property for spaces of holomorphic functions on infinite dimensional spaces I, J. Approx. Theory 126 (2004), 141-156.

[14] K. Floret and J. Wloka, Einführung in die Theorie der lokalkonvexen Räume, Lecture Notes in Mathematics, No. 56, Springer-Verlag, Berlin, 1968.

[15] P. Galindo, D. García, and M. Maestre, Holomorphic mappings of bounded type, J. Math. Anal. Appl. 166 (1992), 236-246.

[16] A. Grothendieck, Produits tensoriels topologiques et espaces nucléaires, Mem. Amer. Math. Soc. 16 (1955), 140.

[17] J. Horváth, Topological vector spaces and distributions. Vol. I, Addison-Wesley Publishing Co., Reading, Mass.-London-Don Mills, Ont., 1966.

[18] H. Jarchow, Locally convex spaces, B. G. Teubner, Stuttgart, 1981, ISBN 3-519-02224-9.

[19] G. Köthe, Topological vector spaces. I, Grundlehren der mathematischen Wissenschaften, vol. 159, Springer-Verlag New York Inc., New York, 1969.

[20] - Topological vector spaces. II, Grundlehren der Mathematischen Wissenschaften, vol. 237, Springer-Verlag, New York, 1979, ISBN 0-387-90400-X. 
[21] J. Lindenstrauss and L. Tzafriri, Classical Banach spaces. I, Springer-Verlag, Berlin, 1977, ISBN 3-540-08072-4.

[22] P. Mazet, Analytic sets in locally convex spaces, North-Holland Mathematics Studies, vol. 89, North-Holland Publishing Co., Amsterdam, 1984, ISBN 0-444-86867-4.

[23] R. Meise, Räume holomorpher Vektorfunktionen mit Wachstumsbedingungen und topologische Tensorprodukte, Math. Ann. 199 (1972), 293-312.

[24] J. Mujica, Domains of holomorphy in (DFC)-spaces, Functional Analysis, Holomorphy, and Approximation Theory (Proc. Sem., Univ. Fed. Rio de Janeiro, Rio de Janeiro, 1978), Lecture Notes in Math., vol. 843, Springer, Berlin, 1981, pp. 500-533.

[25] Complex analysis in Banach spaces, North-Holland Mathematics Studies, vol. 120, North-Holland Publishing Co., Amsterdam, 1986, ISBN 0-444-87886-6.

[26] Linearization of bounded holomorphic mappings on Banach spaces, Trans. Amer. Math. Soc. 324 (1991), 867-887.

[27] Linearization of holomorphic mappings of bounded type, Progress in Functional Analysis (Peñíscola, 1990), North-Holland Math. Stud., vol. 170, North-Holland, Amsterdam, 1992, pp. 149-162.

[28] J. Mujica and L. Nachbin, Linearization of holomorphic mappings on locally convex spaces, J. Math. Pures Appl. (9) 71 (1992), 543-560.

[29] R. Ryan, Applications of topological tensor products to infinite dimensional holomorphy, Dublin, 1980.

[30] M. Schottenloher, $\varepsilon$-product and continuation of analytic mappings, Analyse Fonctionnelle Et Applications (Comptes Rendus Colloq. d'Analyse, Inst. Mat., Univ. Federal Rio de Janeiro, Rio de Janeiro, 1972), Hermann, Paris, 1975, pp. 261-270. Actualités Sci. Indust., No. 1367.

[31] L. Schwartz, Produits tensoriels topologiques d'espaces vectoriels topologiques. Espaces vectoriels topologiques nucléaires. Applications., Séminaire Schwartz, 1953/1954.

[32] _ Théorie des distributions à valeurs vectorielles. I, Ann. Inst. Fourier, Grenoble 7 (1957), 1-141. 